\title{
Synthetic Aperture Radar Systems for Small Aircrafts: Data Processing Approaches
}

\author{
Oleksandr O. Bezvesilniy and Dmytro M. Vavriv \\ Institute of Radio Astronomy of the National Academy of Sciences of Ukraine
}

Ukraine

\section{Introduction}

The synthetic aperture radar (SAR) is considered now as the most effective instrument for producing radar images of ground scenes with a high spatial resolution. The usage of small aircrafts as the platform for the deployment of SAR systems is attractive from the point of view of many practical applications. Firstly, this enables for a substantial lowering of the exploitation costs of SAR sensors. Secondly, such solution provides a possibility to perform a rather quick surveillance and imaging of particular ground areas. Finally, the progress in this direction will allow for a much wider application of SAR sensors.

However, the formation of high-quality SAR images with SAR systems deployed on small aircrafts is still a challenging problem. The main difficulties come from significant variations of the aircraft trajectory and the antenna orientation during real flights. These motion errors lead to defocusing, geometric distortions, and radiometric errors in SAR images.

In this chapter, we describe three effective approaches to the SAR data processing, which enable the solution of the above problems:

1. Time-domain SAR processing with clutter-lock and geometric correction by resampling,

2. Time-domain SAR processing with built-in geometric correction and multi-look radiometric correction,

3. Range-Doppler algorithm with the 1-st and 2-nd order motion compensation.

The proposed solutions have been successfully implemented in $\mathrm{Ku}$ - and X-band SAR systems developed and produced at the Institute of Radio Astronomy of the National Academy of Sciences of Ukraine. The efficiency of the proposed algorithms is illustrated by SAR images obtained with these SAR systems.

The chapter is organized as follows. In Section 2, basic principles of SAR data processing is described. In Section 3, the problem of motion errors of airborne SAR systems is considered, and the appearance of geometric distortions and radiometric errors in SAR images is discussed. The three data processing approaches are considered in details in Sections 4, 5, and 6. Section 7 describes the RIAN-SAR-Ku and RIAN-SAR-X systems used in our experiments. The conclusion is given in Section 8 . 


\section{Principles of SAR data processing}

The synthetic aperture technique is used to obtain high-resolution images of ground surfaces by using a radar with a small antenna installed on an aircraft or a satellite. The radar pulses backscattered from a ground surface and received by the moving antenna can be considered as the pulses received by a set of antennas distributed along the flight trajectory. By coherent processing of these pulses it is possible to build a long virtual antenna - the synthetic aperture that provides a high cross-range resolution. A high range resolution is typically achieved by means of a pulse compression technique that involves transmitting long pulses with a linear frequency modulation or a phase codding.

\subsection{Concept of the synthetic aperture}

Practical SAR systems are produced to operate in one or several operating modes. Depending on the mode, they are referred as the strip-map SAR, the spot-light SAR, the inverse SAR, the ScanSAR, and the interferometric SAR (Bamler \& Hartl, 1998; Carrara at al., 1995; Cumming \& Wong, 2005; Franceschetti \& Lanari, 1999; Rosen at al., 2000; Wehner, 1995). We shall consider mainly the most popular and practically useful strip-map SAR operating mode. However, the presented further results are applicable to other modes to a large extent.

In the strip-map SAR mode, the radar performs imaging of a strip on the ground aside of the flight trajectory. Geometry of the strip-map mode is shown in Fig. 1. The aircraft flies along the straight line above the $x$-axis with the velocity $V$ at the altitude $H$ above the ground plane $(x y)$.

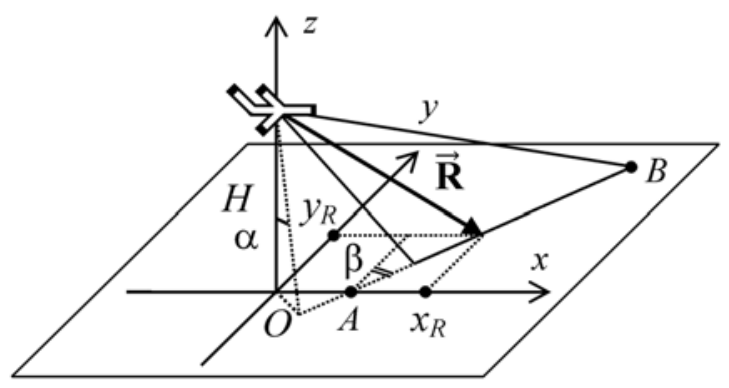

Fig. 1. Geometry of the strip-map SAR mode.

The orientation of the real antenna beam is described by the pitch angle $\alpha$ and the yaw angle $\beta$ that are measured with respect to the flight direction. The line $A B$ in Fig. 1 is the intersection of the elevation plane of the real antenna pattern and the ground plane. This line is called the Doppler centroid line. The coordinates of the point $\left(x_{R}, y_{R}\right)$ on this line at the slant range $R$ from the aircraft are given by

$$
x_{R}=H \tan \alpha \cos \beta+\sin \beta \sqrt{R^{2}-H^{2}-(H \tan \alpha)^{2}},
$$




$$
y_{R}=-H \tan \alpha \sin \beta+\cos \beta \sqrt{R^{2}-H^{2}-(H \tan \alpha)^{2}} .
$$

In order to form the synthetic aperture and direct the synthetic beam to the point $\left(x_{R}, y_{R}\right)$, the signal $s_{R}(\tau+t)$ backscattered from this point should be summed up coherently on the interval of synthesis $-T_{S} / 2 \leq \tau \leq T_{S} / 2$ taking into account the propagation phase $\varphi(\tau)$ (Cumming \& Wong, 2005; Franceschetti \& Lanari, 1999):

$$
\begin{gathered}
I\left(t, x_{R}, y_{R}\right)=\left|\frac{1}{T_{S}} \int_{-T_{S} / 2}^{T_{S} / 2} s_{R}(\tau+t) h_{R}(\tau) d \tau\right|^{2}, \\
h_{R}(\tau)=w_{R}(\tau) \exp [-i \varphi(\tau)], \quad \varphi(\tau)=-\frac{4 \pi}{\lambda} R(\tau) .
\end{gathered}
$$

Here $I\left(t, x_{R}, y_{R}\right)$ is the SAR image pixel, $t$ is the time when the aircraft is at the centre of the synthetic aperture $(0,0, H), \tau$ is the time within the interval of synthesis, $h_{R}(\tau)$ is the azimuth reference function in the time domain, $w_{R}(\tau)$ is the weighting window applied to improve the side-lobe level of the synthetic aperture pattern, $\lambda$ is the radar wavelength, and $R(\tau)$ is the slant range to the point:

$$
R(\tau)=\sqrt{\left(x_{R}-V \tau\right)^{2}+y_{R}^{2}+H^{2}}=\sqrt{R^{2}-2 x_{R} V \tau+(V \tau)^{2}} .
$$

If the slant range $R(\tau)$ changes during the time of synthesis $T_{S}$ more than the size of the range resolution cell, then the target signal "migrates" through several range cells. This effect known as the range migration should be taken into account during the aperture synthesis. The one-dimensional backscattered signal $s_{R}(\tau+t)$ should be obtained from the two-dimensional "azimuth - slant range" matrix of the range-compressed radar data by the interpolation along the migration curve (5).

The instant Doppler frequency of the received signal is, approximately,

$$
f(\tau)=-\frac{2}{\lambda} \frac{d R(\tau)}{d t} \approx F_{D C}+F_{D R} \tau
$$

where the Doppler centroid $F_{D C}$ and the Doppler rate $F_{D R}$ are given by

$$
\begin{gathered}
F_{D C}=\frac{2}{\lambda} V \frac{x_{R}}{R}, \\
F_{D R}=-\frac{2}{\lambda} \frac{V^{2}}{R}\left[1-\left(\frac{x_{R}}{R}\right)^{2}\right] .
\end{gathered}
$$

It is useful to note that the Doppler centroid determines the synthetic beam direction, whereas the Doppler rate is responsible for the beam focusing.

From the point of view of signal processing, the formation of the synthetic aperture (3) is the matched filtering of linear frequency modulated signals (6). Such filtering can be performed 
either in the time or in the frequency domain. Accordingly, there are time- and frequencydomain SAR processing algorithms.

It is easy to show that the azimuth resolution $\rho_{X}$ is given by (Cumming \& Wong, 2005;

Carrara at al., 1995)

$$
\rho_{X}=K_{w} \frac{V}{\Delta F_{D}}
$$

Here $\Delta F_{D}=\left|F_{D R}\right| T_{S}$ is the Doppler frequency bandwidth that corresponds to the interval of the synthesis. The coefficient $K_{w}$ describes the broadening of the main lobe of the synthetic aperture pattern caused by windowing.

In order to improve the quality of SAR images, a multi-look processing technique is used in most modern SAR systems (Moreira, 1991; Oliver \& Quegan, 1998). According to such technique, a long synthetic aperture is divided on shorter intervals that are processed independently to build several SAR images of the same ground scene, called SAR looks. It can be considered as building the synthetic aperture with multiple synthetic beams. A non-coherent averaging of the SAR looks into one multi-look image is used to reduce speckle noise and to reveal fine details in SAR images. Multi-look processing can be used for other applications, for example, for measuring the Doppler centroid with a high accuracy and high spatial resolution and retrieving 3D topography of ground surfaces (Bezvesilniy et al., 2006; Bezvesilniy et al., 2007; Bezvesilniy et al., 2008; Vavriv \& Bezvesilniy, 2011b).

In the next sections we consider peculiarities of the realization of SAR processing algorithms in time and frequency domains.

\subsection{SAR processing in time domain}

The SAR processing in the time domain is performed according to the relations (3)-(5). The block-scheme of the algorithm is shown in Fig. 2.

The received range-compressed radar data are stored in a memory buffer. The buffer size in the range corresponds to the swath width; the buffer size in the azimuth is determined by the time of synthesis. The basic step of the SAR processing procedure for a given range $R$ includes the following calculations:

1. Calculation of the Doppler centroid (7), the Doppler rate (8), and the required time of synthesis (9),

2. Interpolation along the migration curve (5),

3. Multiplication by the reference function with windowing (4), and

4. Coherent summation (3).

As the result, a single pixel of the SAR image is obtained representing the ground point on the Doppler centroid line at the range $R$. This basic step is repeated for all ranges within the swath producing a single line of the SAR image in the range direction. In order to form the next line of the SAR image, the data in the buffer is shifted in the azimuth and supplemented with new data, and the computations are repeated. 


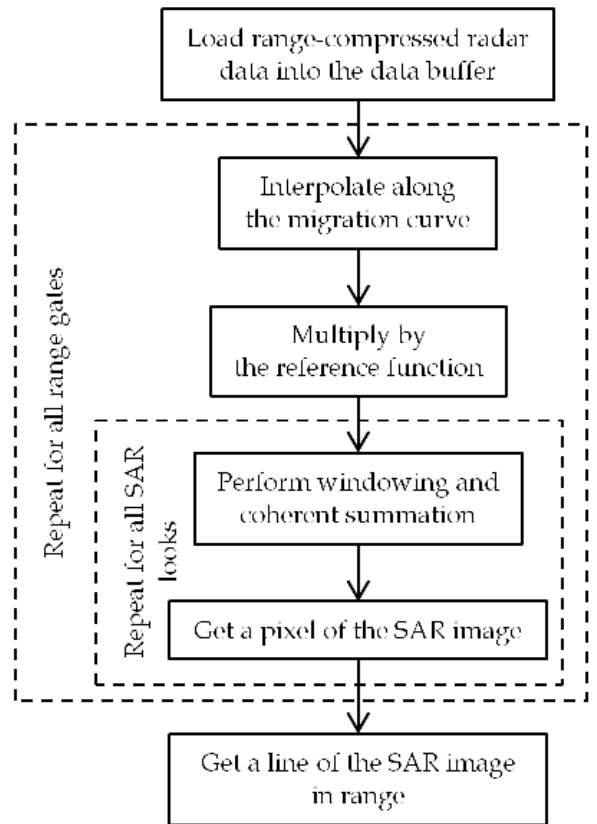

Fig. 2. SAR processing in time domain.

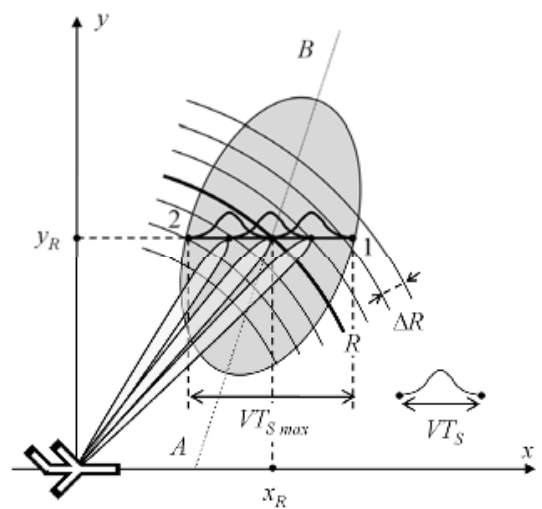

(a)

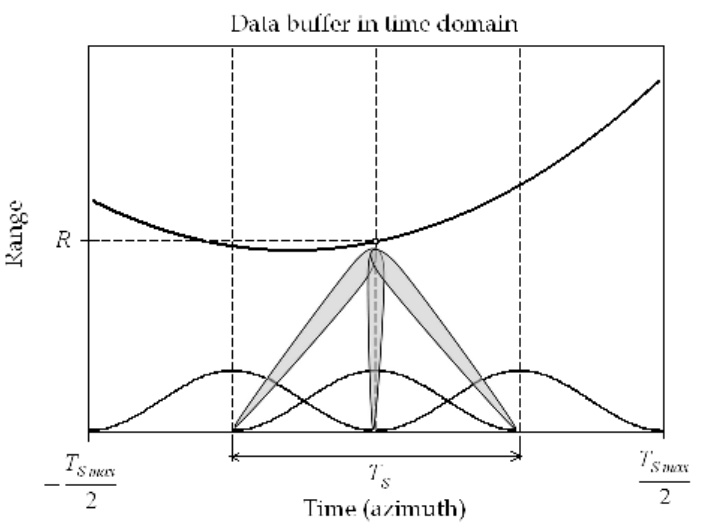

(b)

Fig. 3. Multi-look processing in the time domain: (a) the antenna footprint consideration, (b) the data buffer consideration.

The multi-look processing in the time domain is usually performed directly following the definition (Moreira, 1991; Oliver \& Quegan, 1998). Namely, the reference functions and range migration curves are built for the long interval of synthesis $T_{S \max }$, which is the time required for the ground target to cross the antenna footprint from point 1 to point 2 in Fig. 
3a. The multi-look processing is performed by splitting the long interval of the synthesis $T_{S \max }$ on several sub-intervals $T_{S}$, forming in this manner multiple synthetic beams pointed to the same point on the ground at the different moments of time, as shown in Fig. 3b. The number of the looks for a scheme with the half-overlapped sub-intervals is given by

$$
N_{L}=\operatorname{int}\left\{\frac{T_{S \max }}{T_{S} / 2}\right\}-1
$$

\subsection{SAR processing in frequency domain}

The SAR data processing can be also performed effectively in the frequency domain. It is known that the convolution of two signals in the time domain is equivalent to the multiplication of their Fourier pairs in the frequency domain. The corresponding computations are efficient due to the application of the fast Fourier transform (FFT). A number of FFT-based SAR processing algorithms have been so far developed (Cumming \& Wong, 2005).

In particular, the range-Doppler algorithm (RDA) (Cumming \& Wong, 2005) is a relatively simple and widely-used FFT-based algorithm. The processing steps of this algorithm are shown in Fig. 4 and illustrated also in Fig. 5. The received radar data are stored in a large memory buffer. The buffer size in the range direction corresponds to the swath width, and the buffer size in the azimuth direction is equal to the length of the FFT that covers many intervals of synthesis. First, the range-compressed data are transformed into the rangeDoppler domain by applying FFT in the azimuth. The frequency scale is limited by the pulse repetition frequency (PRF). Then, the range migration correction is performed in the frequency domain. By using the relation (6) between the instant frequency $f$ and the time $\tau$ within the interval of synthesis (preserving the square-root law for the slant range) one can derive the formula for the migration curve in the frequency domain from the migration curve (5) in the time domain:

$$
R(f)=R \sqrt{1-\frac{\lambda^{2} F_{D C}^{2}}{4 V^{2}}} / \sqrt{1-\frac{\lambda^{2} f^{2}}{4 V^{2}}} .
$$

After that, the phase compensation and windowing are applied for the azimuth compression. By using the principle of stationary phase (Cumming \& Wong, 2005) an expression for the reference function in the frequency domain is obtained:

$$
h_{R}(f)=w_{R}(f) \exp [-i \theta(f)], \theta(f)=-\frac{4 \pi}{\lambda} R\left[\sqrt{1-\left(\frac{\lambda f}{2 V}\right)^{2}} \sqrt{1-\left(\frac{\lambda F_{D C}}{2 V}\right)^{2}}+\left(\frac{\lambda f}{2 V}\right)\left(\frac{\lambda F_{D C}}{2 V}\right)\right] .
$$

Finally, the SAR image is formed by applying the inverse FFT in the azimuth. Thus, the basic processing step in the frequency domain performed for a given range gives the line of the SAR image in the azimuth. This basic step is repeated for all ranges within the swath producing the complete SAR image of the ground scene presented in the data frame. 


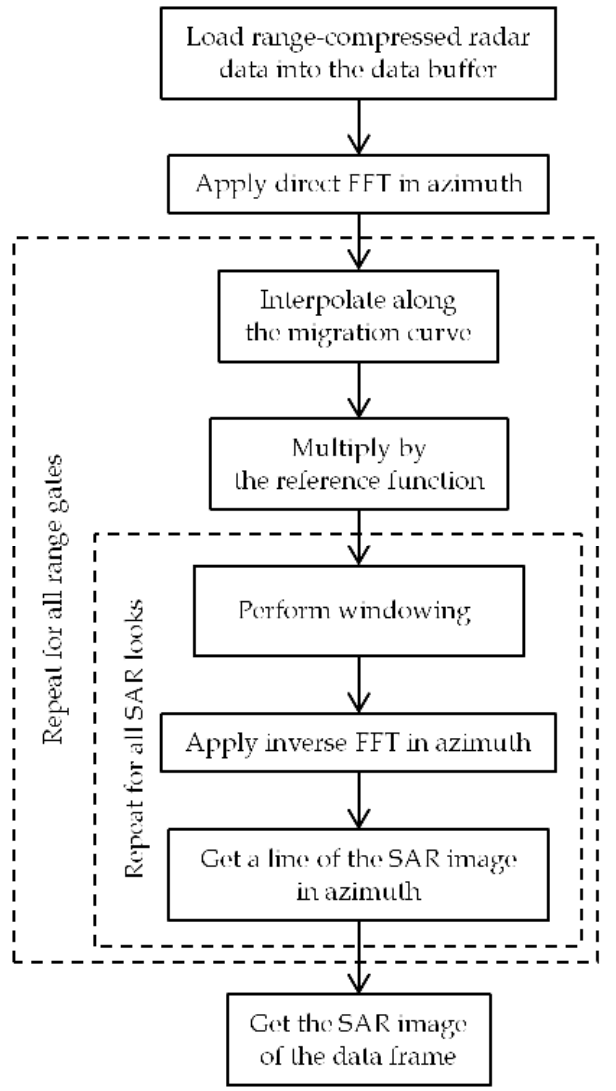

Fig. 4. SAR processing in frequency domain.

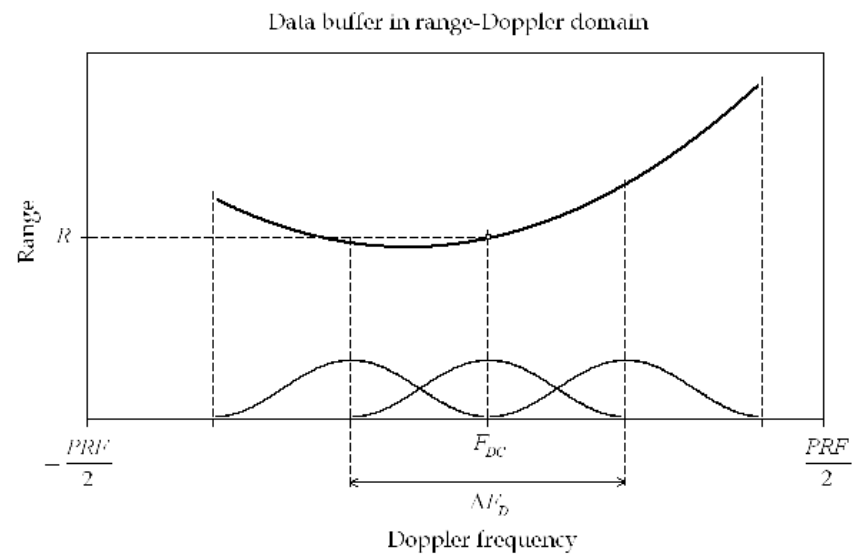

Fig. 5. Multi-look processing in frequency domain. 
In the cases of a significantly squinted geometry (a large antenna yaw angle) or a very high resolution, or a large number of looks, an additional processing step called "secondary range compression" is required (Cumming \& Wong, 2005).

The multi-look processing is performed in the frequency domain by dividing the whole Doppler band $\Delta F_{D \max }=\left|F_{D R}\right| T_{S \max }$ of the backscattered radar signals into the sub-bands $\Delta F_{D}$ for the separate azimuth compression (Cumming \& Wong, 2005; Carrara at al., 1995):

$$
\Delta F_{D}=\frac{\Delta F_{D \max }}{\left(N_{L}+1\right) / 2}
$$

For the multi-look processing scheme with the half-overlapped sub-bands, the central frequencies of the SAR looks with respect to the Doppler centroid are given by

$$
\Delta F_{C}\left(R, n_{L}\right)=F_{D C}(R)-n_{L} \frac{\Delta F_{D}}{2},
$$

where $n_{L}=-N_{L} / 2, \ldots, N_{L} / 2-1$ is the SAR look index. Since the Doppler rate (8) is always negative, the first sub-interval in the time domain corresponds to the last sub-band in the frequency domain. Therefore, we write the minus sign in (14).

\section{Problem of aircraft motion errors}

Deviations of the aircraft flight trajectory and instabilities of the aircraft orientation significantly complicate the formation of SAR images. Such motion errors lead to defocusing, geometric distortions, and radiometric errors in SAR images (Blacknell et al., 1989; Buckreuss, 1991; Franceschetti \& Lanari, 1999; Oliver \& Quegan, 1998). In this section, we shall discuss these problems and their solutions in details.

\subsection{Aircraft flight with motion errors}

The trajectory of an aircraft may deviate from a straight line significantly in real flights. The orientation of the aircraft could also be unstable. These motion errors should be measured and compensated in order to produce high-quality SAR images. We assume that the navigation system is capable of measuring the aircraft trajectory and the aircraft velocity vector. We suppose also that the orientation of the real antenna beam with respect to the velocity vector is known.

Usually, the final product of the strip-map SAR system is a sequence of SAR images of a particular dimension, built in a projection to the ground plane, with indication of the north direction and the latitude-longitude position. Later, if necessary, several consequent images can be stitched together to produce a larger map of a particular ground area of interest. Thus, the received radar data is processed by data frames. Each frame gives one SAR image from the image sequence. The data frames are usually overlapped to guarantee successful stitching of the produced SAR images without gaps.

In order to produce the SAR image from the data frame, it is needed to define a reference flight line for this frame, the averaged flight altitude $H_{\text {ref }}$ and the averaged velocity $V_{\text {ref }}$. Under unstable flight conditions, the reference flight line should be close to the actual 
curvilinear flight trajectory of the aircraft. Also, the reference antenna pitch and yaw angles $\alpha_{r e f}$ and $\beta_{r e f}$, which describes the averaged orientation of the real antenna beam during the time of the data frame acquisition, should be introduced.

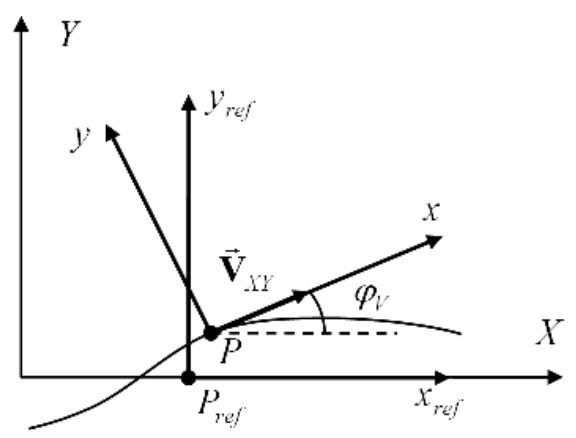

Fig. 6. The scene coordinate system, the reference local coordinate system, and the actual local coordinate system.

Let us define the scene coordinate system $(X, Y, Z)$ so that the reference flight line goes exactly above the $X$ axis. The final-product SAR image is to be sampled on the coordinate grid of the ground plane $(X, Y)$ of this coordinate system. The scene coordinate system is shown in Fig. 6 together with the actual local coordinate system $(x, y, z)$, which slides along the real aircraft flight trajectory, and the reference local coordinate system $\left(x_{\text {ref }}, y_{\text {ref }}, z_{\text {ref }}\right)$, which slides along the $X$ axis (that is along the reference flight line). The current flight direction is described by the angle $\varphi_{V}$ between the horizontal component of the velocity vector $\overrightarrow{\mathbf{V}}_{X Y}$ and the $X$ axis.

The aircraft trajectory $\left(X_{A}(t), Y_{A}(t), Z_{A}(t)\right)$ is described in the scene coordinate system. The actual local coordinates $(x, y)$ and the reference local coordinates $\left(x_{\text {ref }}, y_{\text {ref }}\right)$ are related to each other as follows:

$$
\begin{gathered}
x=\left[x_{r e f}-X_{A}(t)+V_{r e f} t\right] \cos \varphi_{V}(t)+\left[y_{r e f}-Y_{A}(t)\right] \sin \varphi_{V}(t), \\
y=-\left[x_{r e f}-X_{A}(t)+V_{r e f} t\right] \sin \varphi_{V}(t)+\left[y_{r e f}-Y_{A}(t)\right] \cos \varphi_{V}(t)
\end{gathered}
$$

The pitch $\alpha(t)$ and yaw $\beta(t)$ angles describe the antenna beam orientation with respect to the current aircraft velocity vector or, in other words, with respect to the actual local coordinate system. It means that when the synthetic beam is directed to the point $\left(x_{R}, y_{R}\right)$ on the Doppler centroid line by using the Doppler centroid (7), the Doppler rate (8), and the migration curve (5) under unstable flight conditions, the coordinates $\left(x_{R}, y_{R}\right)$ are given in the actual local coordinate system. In order to find the scene coordinates (or the reference local coordinates) of this point, the above relations (15), (16) should be used.

An example of motion errors typical for a light-weight aircraft AN-2 is shown in Fig. 7. In the figure, one can see the coordinate grid of the radar coordinates "slant range - azimuth" 
projected onto the ground plane $(X, Y)$. The horizontal curves are the curves of the constant slant range from the aircraft. They are curved because of deviations of the trajectory from the straight line. The vertical lines are the central lines of the antenna footprint (the Doppler centroid lines) for the consequent aircraft positions. As it is seen, the central lines are not equidistant and not parallel because of variations of the antenna orientation.

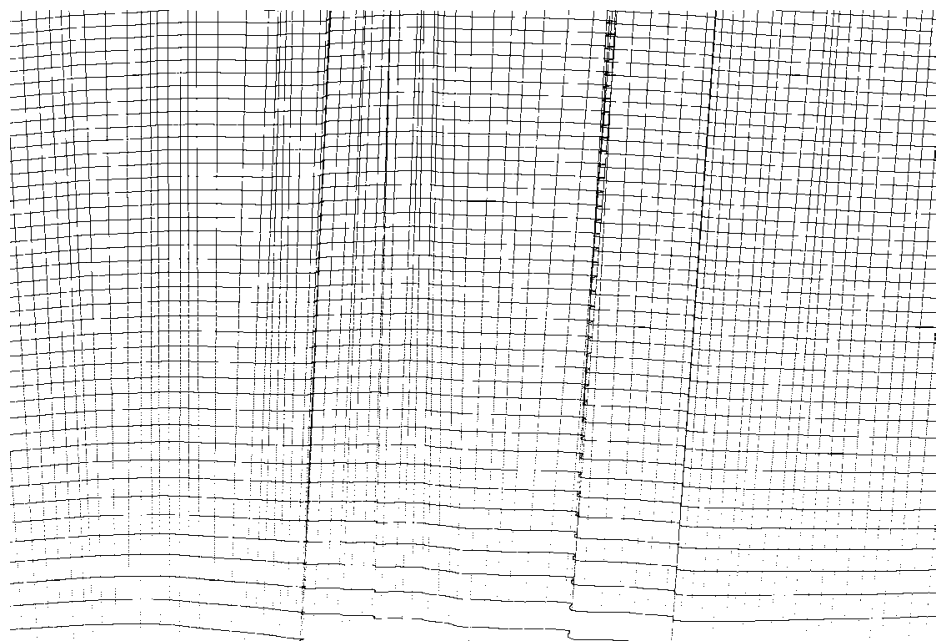

Fig. 7. Trajectory deviations and orientation instabilities illustrated by the coordinate grid in the radar coordinates "slant range - azimuth" on the ground plane.

\subsection{Geometric distortions in SAR images}

The direction of the synthetic beam is determined by the used Doppler centroid with respect to the current velocity vector. In other words, the Doppler centroid controls the direction of the synthetic beam with respect to the actual local coordinate system. Therefore, if the deflections of the velocity vector from the reference flight direction (described by the angle $\varphi_{V}$ in Fig. 6) are not compensated properly, then the synthetic beam is moving forward or backward along the flight path with respect to the scene coordinate system. It means that the scene will be sampled non-uniformly in the azimuth direction resulting in geometric distortions in SAR images. For example, if the synthetic beams are pointed to the centre of the real beam, i.e. to the Doppler centroid line, then the scene will be sampled on a nonuniform grid like that shown in Fig. 7.

If the aircraft trajectory and the orientation of the synthetic aperture beams are known, the geometric distortions can be corrected by resampling of the obtained SAR images to a rectangular grid on the ground plane. This resampling procedure is described in Section 4 . However, this approach could be inefficient in the case of significant geometric distortions.

Alternatively, geometric errors can be avoided if the orientation of the synthetic beams is adjusted at the stage of synthesis by using the trajectory information. The purpose of this adjustment is to keep the beam orientation constant with respect to the reference flight direction. This is the idea of the built-in geometric correction discussed in Section 5. 
The correction of the phase errors and range migration errors caused by trajectory deviations can be applied to the raw data before the aperture synthesis. After such compensation, the raw data look like be collected from the reference straight line. After such motion compensation, the synthetic beams will be set with respect to the reference local coordinate system. Such approach is widely used with the SAR processing algorithms working in the frequency domain. This motion compensation technique is considered in Section 6 with application to the range-Doppler algorithm.

\subsection{Radiometric errors in SAR images}

The problem of radiometric errors is illustrated in Fig. 8. If there are no orientation errors, the synthetic beam of the central look is directed to the centre of the real antenna beam, and all SAR look beams are within the main lobe of the real antenna pattern, as shown in Fig. 8a. The antenna orientation errors lead to the situation when the SAR beams are directed outside the real antenna beam to not-illuminated ground areas, as shown in Fig. 8b, resulting in radiometric errors.
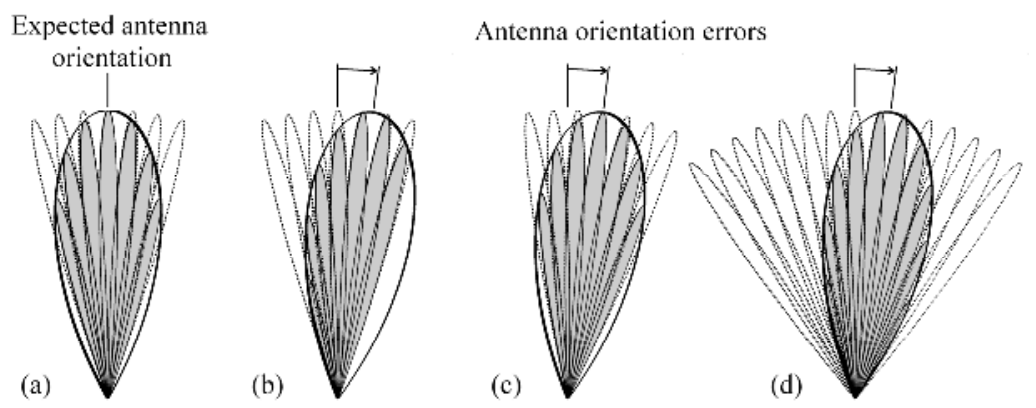

Fig. 8. Multi-look processing without antenna orientation errors (a) and with orientation errors: (b) without clutter-lock, (c) with clutter-lock, (d) with extended number of looks.

Instabilities of the aircraft orientation can be compensated by the antenna stabilization by mounting it on a gimbal. It helps to keep the constant antenna beam orientation. However, this approach is rather complicated and expensive.

The application of a wide-beam antenna firmly mounted on the aircraft is a less expensive way to guarantee a uniform illumination of the ground scene despite of instabilities of the platform orientation. Several shortcomings of this approach should be admitted. The application of a wide antenna beam means some degradation of the radar sensitivity. Also, it calls for a higher PRF to sample the increased Doppler frequency band. Moreover, only the central part of the antenna footprint will be illuminated uniformly limiting the number of looks that can be built without an additional radiometric compensation.

The clutter-lock technique (Li at al., 1985; Madsen, 1989) is usually used to avoid radiometric errors in SAR images. According to the clutter lock technique, the azimuth reference functions are built adaptively so that the synthetic beams track the direction of the real antenna beam staying within the main lobe of the real antenna pattern as shown in Fig. 8c. However, the variations of the synthetic beam orientation due to the clutter-lock naturally lead to geometric distortions in SAR images. 
The clutter lock technique is effective if the variations of the antenna beam orientation are slow in time and small as compared to the real antenna beam width in the azimuth. In this case, the geometric distortions can be corrected by re-sampling. Provided that the orientation instabilities are fast and significant, the clutter-lock leads to strong geometric distortions in SAR images which cannot be easily corrected by re-sampling.

We have proposed an alternative radiometric correction approach, which is based on a multi-look SAR processing with an extended number of SAR looks (Bezvesilniy et al., 2010c; Bezvesilniy et al., 2010d; Bezvesilniy et al., 2011b; Bezvesilniy et al., 2011c). This technique can be used instead of the clutter-lock. The idea of the approach consists in the formation of an extended number of looks to cover directions beyond the main lobe of the real antenna pattern as illustrated in Fig. 8d. In such approach, some of the SAR look beams are always presented within the real antenna beam despite of the orientation errors. In Section 5, we describe how to combine these extended SAR looks to produce the multi-look SAR image without radiometric errors. This approach is appropriate for the cases when the clutter-lock cannot be applied because of fast orientation instabilities or for SAR processing algorithms that cannot be used together with the clutter-lock. The proposed method also allows correcting the radiometric errors in SAR images if the antenna orientation is not known accurately.

\subsection{Dilemma: geometric distortions vs. radiometric errors}

From the above considerations, one can conclude that an attempt to avoid geometric errors by the appropriate pointing of the synthetic beams leads to radiometric errors. And vice versa, the clutter-lock results in geometric errors. So the dilemma of "geometric distortions vs. radiometric errors" should be resolved when developing any SAR data processing approach for SAR systems with motion errors.

We describe three alternative approaches to this problem. In the first approach, described in Section 4, the priority is set to avoiding radiometric errors and the clutter-lock is applied. Geometric errors are corrected by resampling of the obtained SAR images. In the second approach, considered in Section 5, the geometric accuracy of SAR images is the primary goal and we implement a synthetic beam control algorithm called "built-in geometric correction" to point the beams to the nodes of a correct rectangular grid on the ground plane. Radiometric errors are corrected by multi-look processing with extended number of looks. In the third approach, discussed in Section 6, a range-Doppler algorithm with the 1-st and 2nd order motion compensation is considered, which allows obtaining SAR images without significant geometric errors. The application of a wide-beam real antenna could be a solution of the problem of radiometric errors for this approach.

\section{Time-domain SAR processing with clutter-lock and geometric correction by resampling}

In this section, we consider a time-domain SAR data processing algorithm assuming that the aircraft flight altitude and velocity, as well as the antenna beam orientation angles are changed slowly in the sense that they can be considered constant during the time of the synthesis. The main steps of the algorithm are the same as in the case of the straight-line motion with a constant orientation. These steps are described in the block-scheme shown in 
Fig. 2. At each step of the synthesis, the reference function and migration curves are adjusted according to the estimated orientation angles of the real antenna beam providing the clutter-lock. Due to the clutter-lock, it is possible to avoid radiometric errors. Geometric errors are corrected by resampling of the obtained SAR images on the post-processing stage.

\subsection{Estimation of the antenna orientation angles from Doppler centroid measurements}

According to the clutter-lock technique, the synthetic beams are built adaptively to track the direction of the real antenna beam. The orientation angles of the aircraft can be measured by a navigation system. The commonly used navigation systems are based on Inertial Measurement Unit (IMU) or on a combination of IMU and attitude GPS. They are typically rather expensive and do not always provide the required accuracy and the needed rate of measurements. We have proposed an effective method for the estimation of the antenna orientation angles - pitch and yaw - from the Doppler measurements. The application of this technique has allowed us to simplify the navigation system by reducing it to a simple GPS receiver to measure the platform velocity and coordinates only.

The mathematical background of this technique is as follows. The dependence of the Doppler centroid on the slant range is given by

$$
F_{D C}=\frac{2}{\lambda} \frac{(\overrightarrow{\mathbf{R}} \cdot \overrightarrow{\mathbf{V}})}{R}=\frac{2}{\lambda} \frac{x_{R} V_{x}-H V_{z}}{R}
$$

The slant range vector $\overrightarrow{\mathbf{R}}=\left(x_{R}, y_{R},-H\right)$ is directed from the antenna phase centre to the point $\left(x_{R}, y_{R}\right)$ on the Doppler centroid line as shown in Fig. 1, and the aircraft velocity vector is $\overrightarrow{\mathbf{V}}=\left(V_{x}, 0, V_{z}\right)$. In opposite to (7), formula (17) accounts for the possible vertical direction of the aircraft motion. Substituting in (17) the expression (1) for the coordinate of a point on the Doppler centroid line, we rewrite the above dependence as

$$
F_{D C}(R, \alpha, \beta)=\frac{2}{\lambda} \frac{V_{x}}{R}\left[H \tan \alpha \cos \beta+\sin \beta \sqrt{R^{2}-H^{2}-(H \tan \alpha)^{2}}-H \frac{V_{z}}{V_{x}}\right]
$$

The behaviour of the Doppler centroid on range depends strongly on particular values of the antenna pitch and yaw angles as illustrated in Fig. 9. It means that theoretically the antenna beam orientation angles can be estimated via an analysis of the dependence of the measured Doppler centroid on range. However, for the practical implementation of this idea, it was needed to answer the questions: Would it be a reliable estimate? Is it possible to achieve the required accuracy of the angle measurements? And, is it possible to realize this estimation in real time? Fortunately, we have found solutions which provide positive answers on the above questions.

We have found that the pitch and yaw angles can be estimated by fitting the theoretical dependence of the Doppler centroid on range (18) into a set of Doppler centroid values $F_{D C}^{[n]}=F_{D C}\left(R_{n}\right)$ roughly estimated from the received data at each range gate from the Doppler spectra calculated by using the FFT. Here $n$ is the range gate index. 


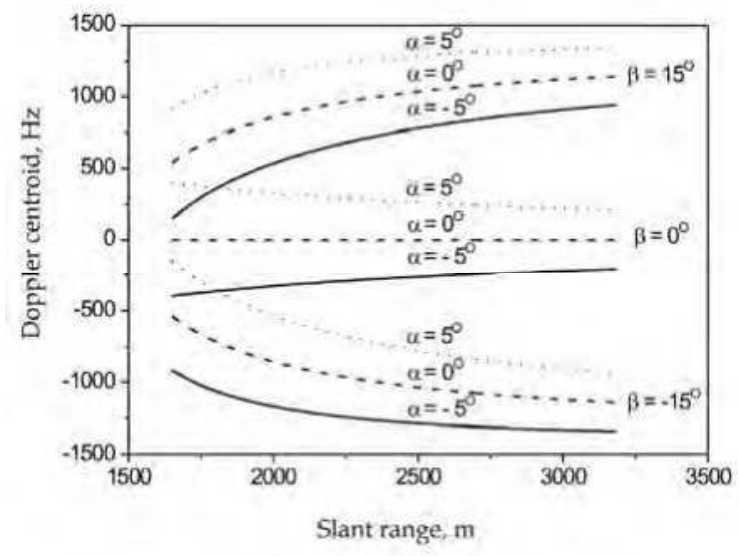

Fig. 9. Dependence of the Doppler centroid on slant range.

We have developed the following fast and effective fitting procedure. By introducing new variables $X_{n}^{i}, Y_{n}$ as

$$
X_{n}^{i}=\sqrt{R_{n}^{2}-H^{2}-\left(H \tan \alpha_{i-1}\right)^{2}}, Y_{n}=\frac{\lambda F_{D C}^{[n]}}{2 V_{x}} R_{n}+H \frac{V_{z}}{V_{x}},
$$

the dependence (18) is transformed into the equation of a straight line:

$$
Y_{n}=\left(H \tan \alpha_{i} \cos \beta_{i}\right)+X_{n}^{i}\left(\sin \beta_{i}\right) .
$$

Thus, the problem of fitting of the non-linear dependence (18) is turned into the well-known task of fitting of a line into a set of experimental points. The only difficulty is that the unknown pitch angle appears in the transformation of the coordinates (19). We have solved this difficulty by using an iteration procedure. The fitting is performed iteratively with respect to the pitch angle considered as a small parameter. The index $i=1,2,3, \ldots$ in (19), (20) is the iteration index. At the first iteration, the pitch angle is assumed to be zero: $\alpha_{0}=0$. It has been found that two iteration are typically enough to achieve the required accuracy of about $0.1^{\circ}$ in real time. The method has been implemented in SAR systems developed and produced at the Institute of Radio Astronomy (Vavriv at al., 2006; Vavriv \& Bezvesilniy, 2011a; Vavriv at al., 2011).

\subsection{Correction of geometric distortions in SAR images by resampling}

In the considered time-domain SAR processing algorithm with the clutter-lock, each line of a SAR image in the range direction represents the ground scene on the Doppler centroid line determined by the current antenna beam orientation angles $\alpha(t)$ and $\beta(t)$ in the actual local coordinate system (see Fig. 6). Thus, the application of the clutter-lock under unstable flight conditions leads to geometric distortions in SAR images as illustrated in Fig. 7. Such geometric distortions can be corrected by resampling of the images from the radar native 
coordinates "slant range - azimuth" to a correct rectangular grid on the ground plane $(X, Y)$ by taking into account the measured aircraft trajectory and the orientation of the synthetic aperture beams.

The resampling procedure consists of the following steps.

1. Define the reference flight line and the reference parameters, as well as the corresponding scene coordinate system for a given SAR image frame as it was described in Section 3.1.

2. Perform the resampling (interpolation) of the SAR image $\operatorname{SAR}\left(X_{A}, R\right)$ from the slant range to the ground range in four steps:

2.1. Calculate the coordinates of the image pixels $\operatorname{SAR}\left(X_{A}, R\right)$ in the actual local coordinate system: $\left(x_{S A R}\left(X_{A}, R\right), y_{S A R}\left(X_{A}, R\right)\right)$.

2.2. Re-calculate the coordinates of the image pixels from the actual local coordinate system to the scene coordinate system according to (15), (16) and obtain the coordinates $\left(X_{S A R}\left(X_{A}, R\right), Y_{S A R}\left(X_{A}, R\right)\right)$.

2.3. Perform a one-dimensional interpolation of the SAR image line-by-line in the range direction from the uniform grid in the slant range to the uniform grid in the ground range. As the result, we obtain the image $\operatorname{SAR}\left(X_{A}, Y\right)$.

2.4. Find the coordinates $X_{S A R}\left(X_{A}, Y\right)$ of the image samples $S A R\left(X_{A}, Y\right)$ in the scene coordinate system from the coordinates $X_{S A R}\left(X_{A}, R\right)$ by the same one-dimensional interpolation in the range direction.

3. Perform the interpolation of the SAR image in the azimuth direction in the following two steps:

3.1 Perform a joint sorting of the pairs of the range-interpolated image samples $\operatorname{SAR}\left(X_{A}, Y\right)$ and their azimuth coordinates $X_{S A R}\left(X_{A}, Y\right)$ in the ascending order with respect to the $X_{A}$-coordinate. This step is required to correct significant forward-backward sweeps of the synthetic beam caused by motion errors.

3.2 Perform a one-dimensional interpolation of the SAR image samples $\operatorname{SAR}\left(X_{A}, Y\right)$ from the initial non-uniform grid of the along-track azimuth coordinate $X_{A}$ to the uniform grid $X$. The result is the desired image $S A R(X, Y)$ in the ground scene coordinates.

The above described resampling algorithm is typically performed as a post-processing procedure.

\subsection{Experimental results}

The described in Sections 4.1 and 4.2 SAR processing approach has been implemented in the airborne RIAN-SAR-Ku system (Vavriv at al., 2006; Vavriv \& Bezvesilniy, 2011a; Vavriv at al., 2011). The light-weight aircrafts Antonov AN-2 and Y-12 were used as the platform.

An example of a single-look SAR image built by the described SAR processing algorithm with the clutter-lock is shown in Fig. 10a. This is the SAR image before the correction of the geometric distortions by resampling. The image resolution is $3 \mathrm{~m}$. The "forward-backward- 


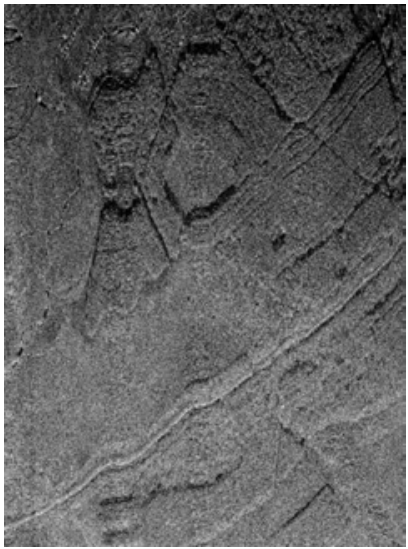

(a)

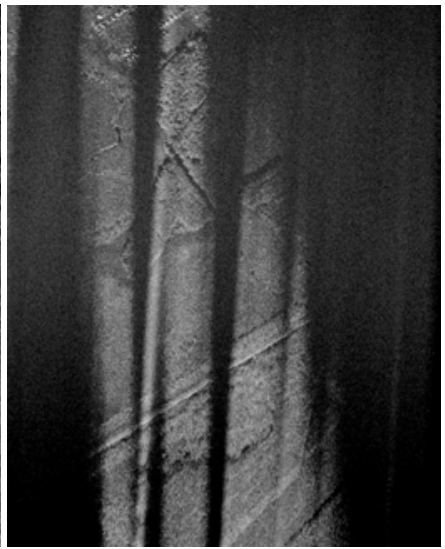

(b)

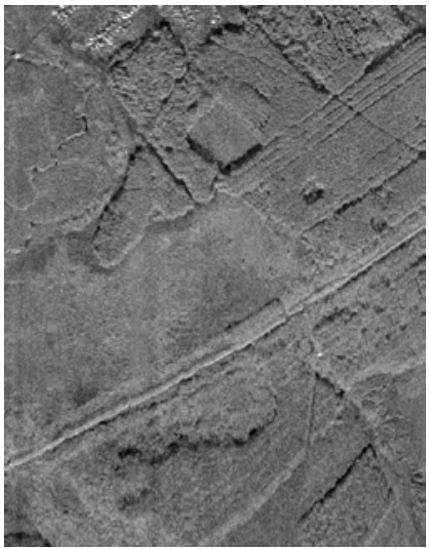

(c)

Fig. 10. Geometric distortions in a single-look SAR image built by using the clutter-lock (a), radiometric errors in the multi-look SAR image built without the clutter lock (b), the multilook SAR image without errors after the resampling procedure (c).

forward" motion of the antenna beam leads to the evident distortions of the road lines and the contours of the forest areas in this image.

A 5-look SAR image formed without the clutter-lock is shown in Fig. 10b. The characteristic amplitude of the antenna beam orientation instabilities was larger than the 1-degree antenna beam width what resulted in significant radiometric errors. It should be noted that the proposed clutter-lock method based on the estimation of the antenna beam from the Doppler centroid measurements is efficient enough to avoid these radiometric errors in Fig. 10a.

The SAR images in Figs. 10a and 10b illustrates the dilemma of "geometric distortions vs. radiometric errors". Radiometric errors are removed due to the clutter-lock in Fig. 10a at the expense of geometric errors. And, vice versa, geometric errors are eliminated in Fig. 10b built without the clutter-lock, but at the cost of significant radiometric errors.

The application of the proposed resampling procedure resolves the dilemma, as it is illustrated in Fig. 10c. In this figure, both geometrical and radiometric errors are corrected.

\section{Time-domain SAR processing with built-in geometric correction and multi- look radiometric correction}

In this section, we describe a SAR processing approach, in which the correction of geometric distortions in SAR images is considered as the primary goal. We proposed (Bezvesilniy et al., 2010a; Bezvesilniy et al., 2010b; Bezvesilniy et al., 2010d; Bezvesilniy et al., 2011a) an algorithm called "built-in geometric correction" to control the synthetic beam direction so that the beams are pointed to the nodes of a correct rectangular grid on the ground plane. As the result, the SAR images are geometrically correct after the synthesis. The synthetic beams are obviously set to the nodes regardless of the real antenna beam orientation. The radiometric errors that arise in this case are corrected by a multi-look processing with extended number of looks. 


\subsection{Multi-look SAR processing on a single-look interval of synthesis}

The multi-look processing in the time domain is usually performed by the coherent processing on sub-intervals of a long interval of the synthesis as described in Section 2.2. According to such approach, it is assumed that there are no significant uncompensated phase errors during the long time of the synthesis $T_{S \max }$ determined by (10). However, as a matter of fact, in order to achieve the desired azimuth resolution it is sufficient to perform the coherent processing on the short time interval $T_{S}$ given by (9). This fact gives an alternative realization of the multi-look processing in the time domain, which is more preferable in the case of significant motion errors. The idea of the algorithm is to process the data collected during the short time of synthesis $T_{S}$ with a set of different reference functions and migration curves to form the SAR look beams. We have called this approach "the multi-look processing on a single-look interval of synthesis". The proposed approach is illustrated in Fig. 11.

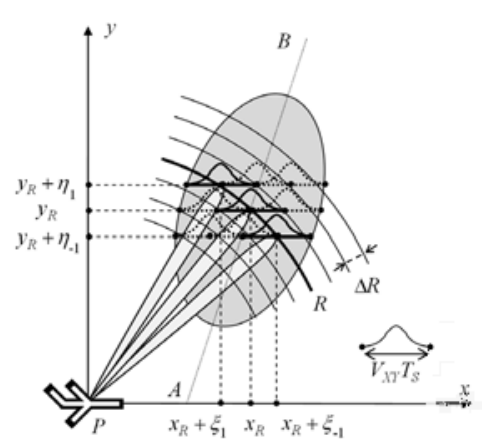

(a)

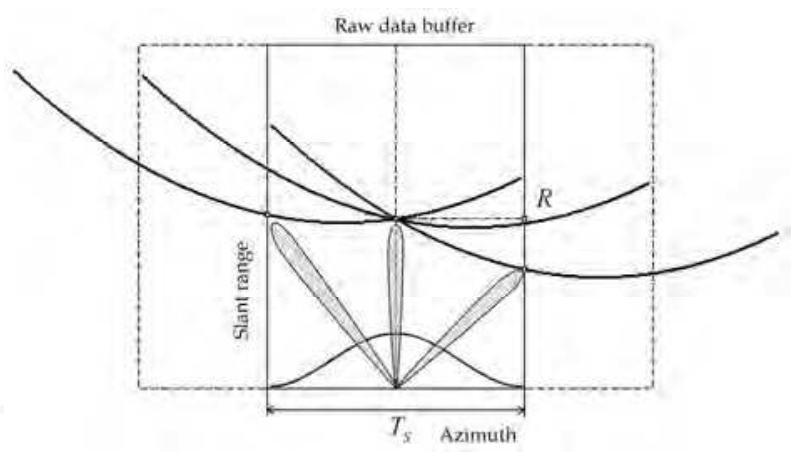

(b)

Fig. 11. The multi-look processing on a single-look interval of synthesis: (a) the antenna footprint consideration, (b) the raw data buffer consideration.

The reference functions of the different SAR looks should be built with the central frequencies (14) similar to the multi-look processing scheme in the frequency domain. The SAR look beam formed with the central frequency $\Delta F_{C}\left(R, n_{L}\right)$ is directed to some point $\left(x_{R}+\xi\left(R, n_{L}\right), y_{R}+\eta\left(R, n_{L}\right)\right)$, which appears at the same slant range $R$ at the centre of the short interval of the synthesis as illustrated in Fig. 11a. Let us derive formulas for these coordinates. The position of the point in the azimuth direction is related to its Doppler centroid (17), so we can write:

$$
\Delta F_{C}\left(R, n_{L}\right)=\frac{2}{\lambda} \frac{\left(x_{R}+\xi\left(R, n_{L}\right)\right) V_{x}-H V_{z}}{R} .
$$

Substituting the expressions (14) and (17) into (21), we obtain:

$$
\xi\left(R, n_{L}\right)=-n_{L} \frac{\lambda R}{2 V_{x}} \frac{\Delta F_{D}}{2} .
$$


Since the points, to which the synthetic beams of the SAR looks are directed, appear at the same slant range at the centre of the short interval of the synthesis, we can write:

$$
x_{R}^{2}+y_{R}^{2}=\left(x_{R}+\xi\left(R, n_{L}\right)\right)^{2}+\left(y_{R}+\eta\left(R, n_{L}\right)\right)^{2},
$$

and, finally,

$$
\eta\left(R, n_{L}\right)=\sqrt{x_{R}^{2}+y_{R}^{2}-\left(x_{R}+\xi\left(R, n_{L}\right)\right)^{2}}-y_{R} .
$$

Thus, in order to form the set of the synthetic beams of the different SAR looks on the short interval of synthesis for the slant range $R\left(n_{L}, X, Y\right)$, we should first calculate the points $\left(x_{R}+\xi\left(R, n_{L}\right), y_{R}+\eta\left(R, n_{L}\right)\right)$ from (22) and (24), which correspond to the required central frequencies (14). Then, we should process the same raw data on the interval of the synthesis $T_{S}$ with the appropriate range migration curves (5), the Doppler centroids (7) and the Doppler rates (8), by substituting the calculated coordinates $\left(x_{R}+\xi\left(R, n_{L}\right), y_{R}+\eta\left(R, n_{L}\right)\right)$ instead of the coordinates $\left(x_{R}, y_{R}\right)$ in these formulas.

The described approach to the multi-look processing has the following benefits. First, it is much easier to keep a low level of the phase errors on the short interval of synthesis, as compared to the long coherent processing time for all looks. Second, the orientation of the real antenna beam does not change significantly during the short processing time. This fact simplifies considerably the calculation of the orientation of all SAR look beams with respect to the real antenna beam for the subsequent radiometric correction. Third, a more accurate motion error compensation can be introduced in this processing scheme as compared to FFT-based algorithms. The compensation is performed based on the measured aircraft trajectory individually for each pixel of the SAR image accounting for both the range and the azimuth dependence of the phase and migration errors without any approximations. In other words, the accuracy of the motion error compensation is limited only by the accuracy of the trajectory measurements.

In the described approach, all SAR look beams are aimed at different points on the ground. It means that the obtained SAR look images are sampled on different grids. Therefore, the SAR look images should be first resampled to the same ground grid and only then they can be averaged to produce the multi-look image. Deviations of the aircraft trajectory introduce further complexity into the re-sampling process. We have proposed (Bezvesilniy et al., 2010a; Bezvesilniy et al., 2010b; Bezvesilniy et al., 2010d; Bezvesilniy et al., 2011a) an algorithm named "the built-in correction of geometric distortions" to solve this problem. This algorithm is described in the next section.

\subsection{Built-in geometric correction}

In order to avoid the interpolation steps in the above-described multi-look processing approach, the reference functions and the migration curves should be specially designed to point the multi-look SAR beams exactly to the nodes of a rectangular grid on the ground plane. The grid nodes to which the multi-look SAR beams should be pointed can be found as follows. The radar data are processed frame-by-frame forming a sequence of overlapped SAR images. For each frame, we define the reference flight line and the reference parameters 
before the synthesis of the aperture. The reference parameters of the data frame are used to calculate the Doppler centroid values $F_{D C}(R)$, the central Doppler frequencies $\Delta F_{C}\left(R, n_{L}\right)$ of the SAR looks, and the coordinates $\left(x_{R}^{r e f}+\xi\left(R, n_{L}\right), y_{R}^{r e f}+\eta\left(R, n_{L}\right)\right)$ of the corresponding points on the ground in the reference local coordinate system. The found points are situated on the central frequency lines, which are similar to the Doppler centroid line $A B$ in Fig. 1. The synthetic beams of the SAR looks should be pointed to the grid nodes, which are closest to the corresponding frequencies lines.

To point the SAR look beam to the found grid node, it is needed to recalculate the coordinates of this node from the reference local coordinate system to the actual local coordinate system by using (15), (16), taking into account the actual aircraft position and the orientation of the aircraft velocity vector. This recalculation is performed at each step of the synthesis. After that, the appropriate range migration curves (7), the Doppler centroids (8), and the Doppler rates (9) can be determined. Finally, the synthetic beam is formed to be directed to this node.

The proposed built-in geometric correction algorithm cannot be combined with the clutterlock technique since the SAR beams do not follow the orientation of the real antenna beam. Therefore, the algorithm works well without an additional radiometric correction only for a wide-beam antenna and only for the central SAR looks. In order to use all possible SAR looks to form a multi-look SAR image without radiometric errors, we have proposed an effective radiometric correction technique based on multi-look processing with extended number of looks (Bezvesilniy et al., 2010c; Bezvesilniy et al., 2010d; Bezvesilniy et al., 2011b; Bezvesilniy et al., 2011c).

\subsection{Radiometric correction by multi-look processing with extended number of looks}

Let us denote an error-free SAR image to be obtained as $I(X, Y)$, where $(X, Y)$ are the ground coordinates of the image pixels. This image is not corrupted by speckle noise and not distorted by radiometric errors. Whereas, a real SAR look image $I\left(n_{L}, X, Y\right)\left(n_{L}\right.$ is the index of the SAR looks) is corrupted by speckle noise $S\left(n_{L}, X, Y\right)$ and distorted by radiometric errors $0<R\left(n_{L}, X, Y\right) \leq 1$ so that

$$
I\left(n_{L}, X, Y\right)=I(X, Y) \cdot S\left(n_{L}, X, Y\right) \cdot R\left(n_{L}, X, Y\right)
$$

The speckle noise in a single-look SAR image (Oliver \& Quegan, 1998) is a multiplicative noise with the exponential probability density function with the mean and the variance, correspondingly,

$$
\mu\left\{S\left(n_{L}, X, Y\right)\right\}=1, \quad \sigma\left\{S\left(n_{L}, X, Y\right)\right\}=1 .
$$

The speckle noise is different for all SAR looks what is indicated here by the SAR look index $n_{L}$. The radiometric errors caused by instabilities of the antenna orientation can be considered as low-frequency multiplicative errors. The highest spatial frequencies of the radiometric error function $R\left(n_{L}, X, Y\right)$ are inversely proportional to the width of the real antenna footprint in the azimuth direction. Similar to the speckle noise, the radiometric errors are different for different SAR looks. 
In order to compensate the radiometric errors, they should be estimated. For this purpose, we use a low-pass filtering $\mathbf{F}$ to measure the local brightness of the SAR images. This filter is designed to pass the radiometric errors and, at the same time, to suppress the speckle noise to some extent:

$$
\mathbf{F}\left\{R\left(n_{L}, X, Y\right)\right\} \approx R\left(n_{L}, X, Y\right), \quad \mathbf{F}\left\{S\left(n_{L}, X, Y\right)\right\} \approx 1 .
$$

The application of this filter to the SAR look image (25) gives, approximately:

$$
I_{L F}\left(n_{L}, X, Y\right)=\mathbf{F}\left\{I\left(n_{L}, X, Y\right)\right\} \approx I_{L F}(X, Y) \cdot R\left(n_{L}, X, Y\right) .
$$

Here $I_{L F}(X, Y)$ is the low-frequency component of the error-free SAR image to be reconstructed. The corresponding components of the real SAR looks $I_{L F}\left(n_{L}, X, Y\right)$ (28) contain information about the radiometric errors and they are almost not corrupted by speckle noise. These images can be used to compare radiometric errors on different SAR looks and, via such comparison, to estimate the radiometric errors. The idea of this empirical approach to the radiometric correction is based on the fact that one of many looks is pointed very closely to the centre of the real antenna beam. This look demonstrates the maximum power (brightness) among all looks, and this power is not distorted by radiometric errors.

Let us denote the number of looks to be summed up into the multi-look image as $N_{L}^{p r o}$. This number of looks is slightly less than the number of the looks within the real antenna beam $N_{L}$ since the orientation instabilities may corrupt the side looks considerably. By using the low-pass filter, it is possible to select the brightest (best-illuminated) parts of the scene among all extended SAR looks with the indexes $n_{L}=1, \ldots, N_{L}^{\text {ext }}$ and compose only $N_{L}^{\text {pro }}$ SAR looks (called the composite looks) for further processing. It is convenient to build the following sequence of the pairs of the composite looks and their low-frequency components:

$$
\left\{I^{\text {pro }}\left(n_{L}^{\text {pro }}, X, Y\right), I_{L F}^{\text {pro }}\left(n_{L}^{\text {pro }}, X, Y\right)\right\}, n_{L}^{\text {pro }}=1, \ldots, N_{L}^{\text {pro }} .
$$

This sequence is kept in the ascending order with respect to the brightness:

$$
I_{L F}^{p r o}\left(n_{L}^{p r o}, X, Y\right) \leq I_{L F}^{p r o}\left(n_{L}^{p r o}+1, X, Y\right) .
$$

After processing of all the extended SAR looks, the brightest composite look is the look with the index $n_{L}^{p r o}=N_{L}^{p r o}$. These brightest values are obtained with the synthetic beams that are directed very closely to the centre of the real beam. Therefore, these brightness values are not distorted by the radiometric errors and give the estimate of the low-frequency component of the error-free SAR image to be reconstructed:

$$
I_{L F}^{p r o}\left(N_{L}^{p r o}, X, Y\right) \approx I_{L F}(x, y) .
$$

This image can be used as the reference to estimate the radiometric error functions for all SAR looks:

$$
R\left(n_{L}, X, Y\right) \approx \frac{I_{L F}\left(n_{L}, X, Y\right)}{I_{L F}^{p r o}\left(N_{L}^{p r o}, X, Y\right)}
$$




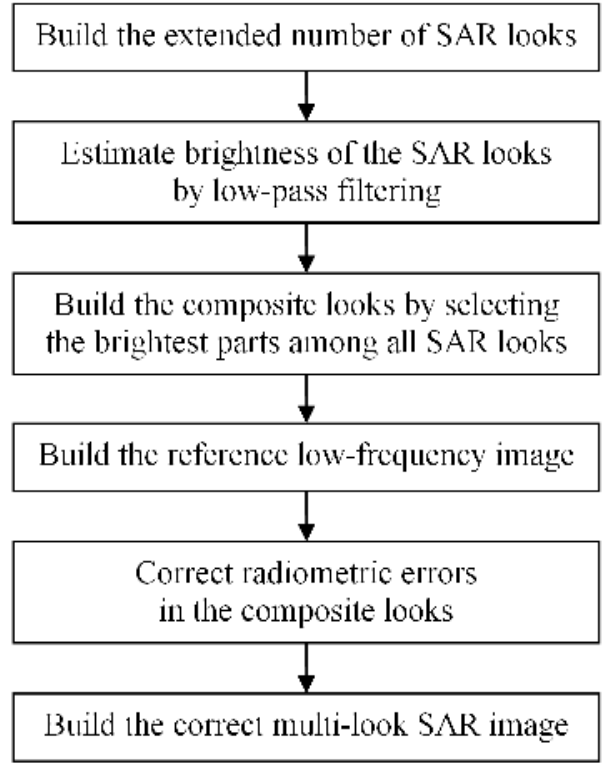

Fig. 12. The main steps of the multi-look radiometric correction algorithm.

By using the estimated radiometric error functions, radiometric errors for all SAR looks can be corrected before combining them into the multi-look SAR image. The main steps of the described algorithm are shown in Fig. 12.

If the navigation system is capable of measuring accurately the fast variations of the real antenna beam orientation, and if the real antenna pattern is known, the radiometric error functions (32) can be calculated directly from the relative orientation of the synthetic beam and the real antenna beam. This approach is more rigorous and accurate than the abovedescribed empirical approach with the image brightness estimation. Nevertheless, with this approach, it is still necessary to build extended number of SAR looks, select the best parts of SAR images among all looks, and form the composite looks for multi-look processing.

\subsection{Experimental results}

The proposed approach has been used for post-processing of the radar data obtained with the RIAN-SAR-Ku and RIAN-SAR-X systems described in Section 7.

The performance of the built-in geometric correction is illustrated in Fig. 13. The SAR image shown in Fig. 13a is built by using the clutter-lock technique. One can see geometric distortions caused by instabilities of the antenna orientation. The undistorted SAR image shown in Fig. 13b is formed by using the algorithm with the built-in geometric correction. Both images have 3-m resolution and are built of 3 looks. The accuracy of the geometric correction is illustrated in Fig. 13c, where the SAR image built of 45 looks and formed by using the built-in geometric correction is imposed on the Google Map image of the scene. 


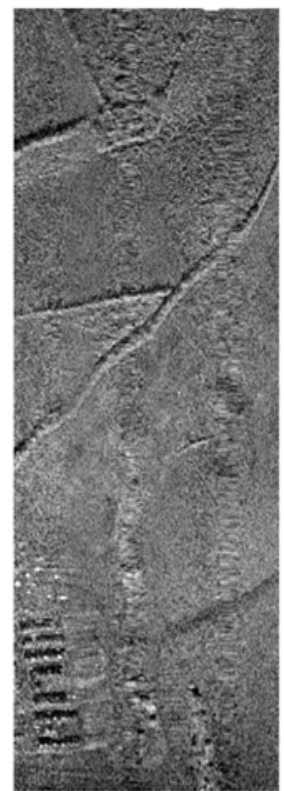

(a)

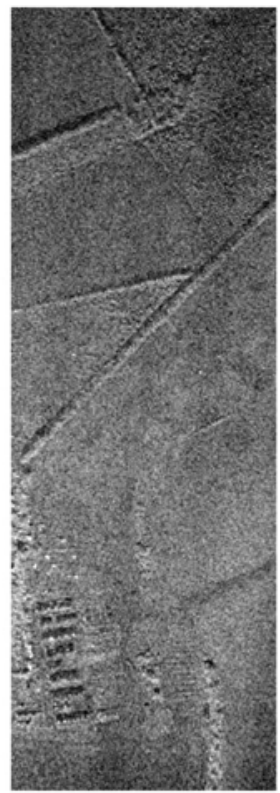

(b)

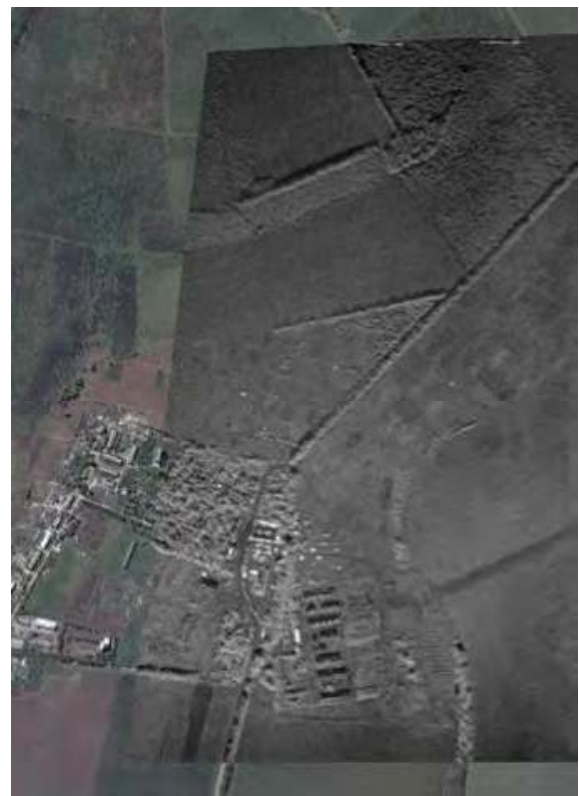

(c)

Fig. 13. Illustration of the geometric correction: (a) the 3-look SAR image built by using the clutter-lock technique, (b) the 3-look SAR image formed by using the built-in geometric correction, and (c) the 45-look SAR image formed by using the built-in geometric correction is imposed on the Google Maps image of the scene.

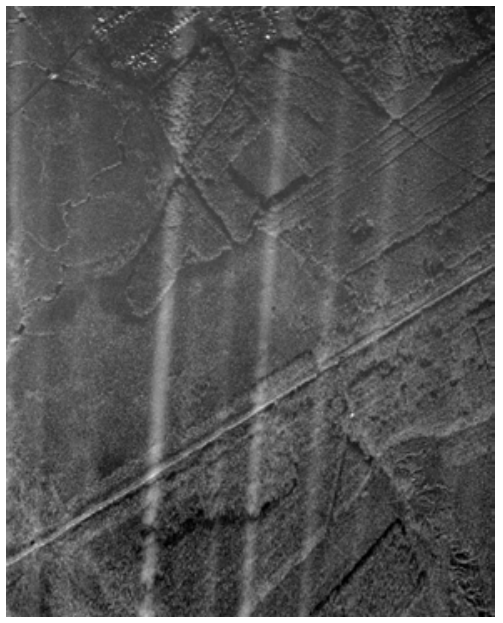

(a)

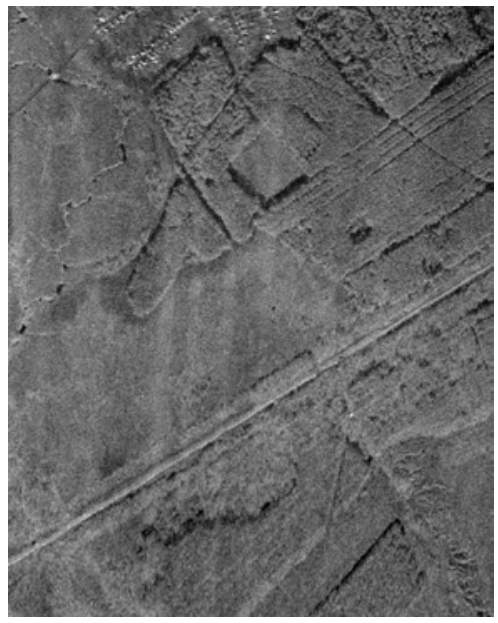

(b)

Fig. 14. Radiometric errors in the SAR image built by simple averaging of all extended SAR looks (a). SAR image formed of 5 composite SAR looks by using the proposed radiometric correction with extended number of looks. 
The performance of the proposed radiometric correction by the multi-look processing with extended number of looks is illustrated in Fig. 14. The SAR image in Fig. 14a is built by simple averaging of all extended SAR looks. The image demonstrates good geometric accuracy; however the radiometric errors are presented. One can see dark and light strips in the image caused by the non-uniform illumination of the scene. The dark areas are due to the illumination for a short time when the real antenna footprint quickly moves to the neighbour areas of the scene. The light areas are correspondently illuminated for a longer time. The SAR image shown in Fig. 14b is built by using the proposed method of the multilook radiometric correction with extended number of looks. The image is built of 5 composite SAR looks. One can see that the radiometric errors have been corrected successfully.

The obtained results prove that the described SAR processing approach can be effectively used for SAR systems installed on light-weight aircrafts with a non-stabilized antenna. An important advantage of the algorithm is that the produced SAR images are already geometrically correct at once after the synthesis, and there is no need in any additional interpolation. Another important advantage of the algorithm is the reduced requirements to the SAR navigation system. Although the aircraft velocity vector should be measured quite accurately to point the synthetic beams at the proper points on the ground, the aircraft trajectory should be measured and compensated with the high accuracy of a fraction of the radar wavelength only during the short time of the synthesis of one look. There is no need to keep so high accuracy of the trajectory measurement during the long time of the data acquisition for all looks.

\section{Range-Doppler algorithm with the 1-st and 2-nd order motion compensation}

The range-Doppler algorithm (RDA) is one of the most popular SAR processing algorithms. A high computational efficiency and a simplicity of the implementation are its main advantages. This algorithm belongs to the frame-based SAR processing algorithms, which use the FFT and work in the frequency domain. The motion compensation within the data frame is required. The SAR images are geometrically correct but they are originally produced in the radar coordinates "slant range - azimuth". Therefore, the ground mapping by an interpolation is required followed by stitching of the obtained image frames into the SAR image of the ground strip. Possible radiometric errors should be additionally corrected.

\subsection{The 1-st and 2-nd order motion compensation}

The geometry of the motion compensation problem is illustrated in Fig. 15. The point $A(0,0, H)$ indicates the expected position of the aircraft on the reference straight line trajectory. The point $A_{E}\left(\Delta x_{E}, \Delta y_{E}, H+\Delta z_{E}\right)$ corresponds to the actual position on the real trajectory. The slant range error for the synthetic beam directed to the point $P\left(x_{R}, y_{R}, 0\right)$ on the Doppler centroid line (1), (2) at the slant range $R$ can be written as

$$
\begin{gathered}
\Delta R_{E}\left(x_{R}, y_{R}\right)=R_{E}\left(x_{R}, y_{R}\right)-R, \\
R_{E}\left(x_{R}, y_{R}\right)=\sqrt{\left(\Delta x_{E}-x_{R}\right)^{2}+\left(\Delta y_{E}-y_{R}\right)^{2}+\left(H+\Delta z_{E}\right)^{2}} .
\end{gathered}
$$


These relations describe both the range migration errors and the corresponding phase errors

$$
\varphi_{E}\left(x_{R}, y_{R}\right)=-\frac{4 \pi}{\lambda} \Delta R_{E}\left(x_{R}, y_{R}\right)
$$

caused by the trajectory deviations $\overrightarrow{\mathbf{r}}_{E}=\left(\Delta x_{E}, \Delta y_{E}, \Delta z_{E}\right)$.

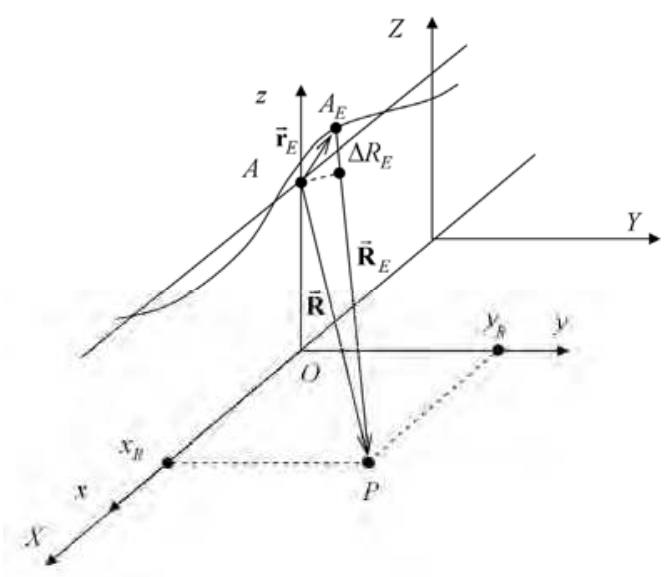

Fig. 15. Geometry of trajectory deviations.

In order to compensate the motion errors, we should correct the range migration errors (33), (34) by introducing an addition interpolation in the range direction and also correct the phase errors (35) in the azimuth direction. The corresponding correction should be performed individually for each pulse on the interval of the synthesis in the accordance with the current aircraft position error $\overrightarrow{\mathbf{r}}_{E}=\left(\Delta x_{E}, \Delta y_{E}, \Delta z_{E}\right)$. The problem is that the range error $\Delta R_{E}$ depends not only on the slant range, but also on the direction to the point $P\left(x_{R}, y_{R}, 0\right)$. It means that the motion errors depend on both range and azimuth and are different for different points on the scene. In other words, the same radar pulses on two overlapped intervals of the synthesis should be compensated individually for the neighbour points in the azimuth direction. Such complete and accurate motion error compensation is possible only in those SAR processing algorithms, which allow the application of an individual reference function and range migration curve for each point of SAR image. It is possible, for example, in the time-domain SAR processing algorithms considered here. However, for the most SAR processing algorithms, including the range-Doppler algorithm, the dependence of the error $\Delta R_{E}$ on the azimuth must be disregarded and the range dependence is taken into account only.

The motion error correction should not interfere with the range and azimuth compression. Any range-dependent motion compensation can not be applied before the range compression of the received radar pulses. Otherwise, the range LFM waveform of the transmitted pulse will be distorted. Also, the range-dependent compensation cannot be applied before the range migration correction step of the SAR processing algorithm. 
Otherwise, different corrections applied for the neighbour range bins will introduce phase errors in azimuth direction.

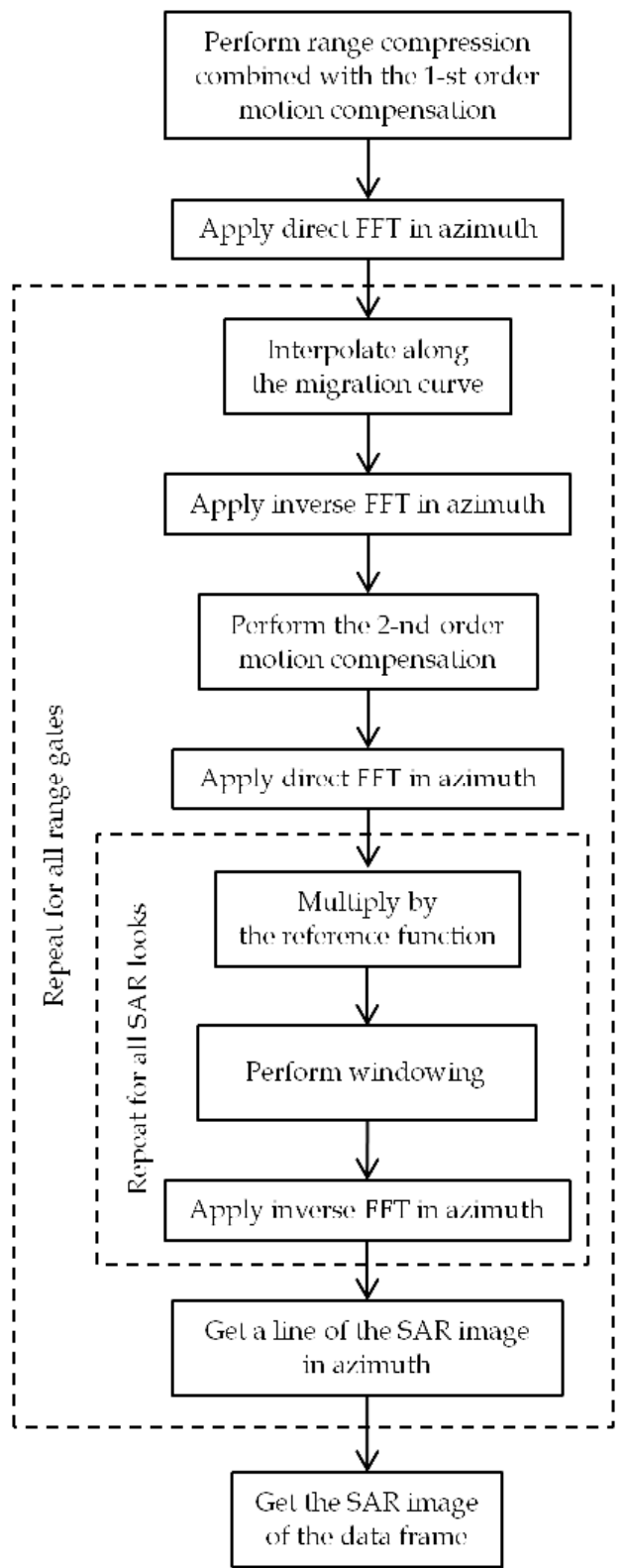

Fig. 16. Range-Doppler algorithm with the 1-st and 2-nd order motion compensation. 
To cope with the above problems, the motion compensation procedure for the rangeDoppler algorithm (and similar FFT-based algorithms) is usually divided on two steps (Franceschetti \& Lanari, 1999):

1. First-order range-independent motion compensation,

2. Second-order range-dependent motion compensation.

The first-order motion compensation includes the range delay (33), (34) of the received pulses (with interpolation) and the phase compensation (35), which are calculated for some reference range, for example, for the centre range of the swath $R_{C}$ :

$$
\varphi_{E}^{(I)}\left(R_{C}, t\right)=\exp \left[-i \frac{4 \pi}{\lambda} \Delta R_{E}^{(I)}\left(R_{C}, t\right)\right] .
$$

Here $t$ is the flight time. The first-order motion compensation can be incorporated into the range compression step but it should be performed before any processing step in the azimuth, in particular, before the range migration correction in the range-Doppler algorithm, as shown in Fig. 16.

The second-order range-dependent motion compensation is performed after the range compression and the range migration correction steps. It includes the phase compensation and may (or may not) include the following range interpolation step:

$$
\begin{aligned}
\Delta R_{E}^{(I I)}(R, t) & =\Delta R_{E}(R, t)-\Delta R_{E}^{(I)}\left(R_{C}, t\right) . \\
\varphi_{E}^{(I I)}(R, t) & =\exp \left[-i \frac{4 \pi}{\lambda} \Delta R_{E}^{(I I)}(R, t)\right] .
\end{aligned}
$$

Since the motion errors depend on time, it is needed to return from the range Doppler domain into the time domain by the inverse FFT, apply the corrections (37), (38), and come back into the range-Doppler domain by applying the direct FFT again, as shown in Fig. 16. After that, we can perform the azimuth compression.

After the compensation, the raw data seem like they are collected from the reference straight line trajectory, and the range-Doppler processing is performed by using the reference parameters of the data frame.

\subsection{Problem of radiometric errors caused by motion compensation}

The RDA performs processing of data blocks in the azimuth frequency domain assuming that the aircraft goes along a straight trajectory with a constant orientation during the time of the data frame accumulation. Therefore, instabilities of the antenna beam orientation within the data frame lead to radiometric errors in SAR images formed by the RDA.

After applying the above-described 1-st and 2-nd order motion compensation procedures, the corrected raw data demonstrate the range migration and phase behaviour as if the data were collected from the reference straight trajectory. However, the illumination of the scene by the real antenna is not changed, and radiometric errors are still presented. 
Moreover, the application of the motion compensation can make the problem of radiometric errors even worse (Bezvesilniy et al., 2011c). After the motion compensation, the location of the antenna footprint on the ground should be described with respect to the position of the aircraft on the reference trajectory by the orientation angles $\alpha_{\mathrm{MoC} o}$ and $\beta_{\mathrm{MoCo}}$ which are different from the angles $\alpha$ and $\beta$ of the actual local coordinate system. The Doppler centroid values of the corrected radar data are apparently different from the Doppler centroid values before the motion compensation. For example, even if the antenna orientation is constant with respect to the actual local coordinate system, the orientation of the antenna beam can demonstrate variations with respect to the reference flight line. It means that the raw data with the constant Doppler centroid could demonstrate Doppler centroid variations after applying the motion compensation. This effect becomes more significant in the case of notably curved trajectories.

The problem of the correction of radiometric errors in SAR images formed by using the range-Doppler algorithm can be solved by the multi-look processing with extended number of looks as it was described in Section 5. It should be pointed out that the clutter-lock based on the estimation of the antenna beam orientation angles from the Doppler centroid measurements can be used together with the range-Doppler algorithm only to estimate the reference orientation angles for each data frame.

\subsection{Experimental results}

The range-Doppler algorithm with the 1-st and 2-nd order motion compensation procedures was implemented in the airborne RIAN-SAR-X system, what allows us to obtain multi-look SAR images in real time. The application of a wide-beam antenna enables avoiding radiometric errors in real time. An example of a 7-look SAR image with a 2-m resolution is given in Fig. 17. The application of the multi-look radiometric correction with the extended number of looks can be applied as a post-processing task to the recorded raw data.

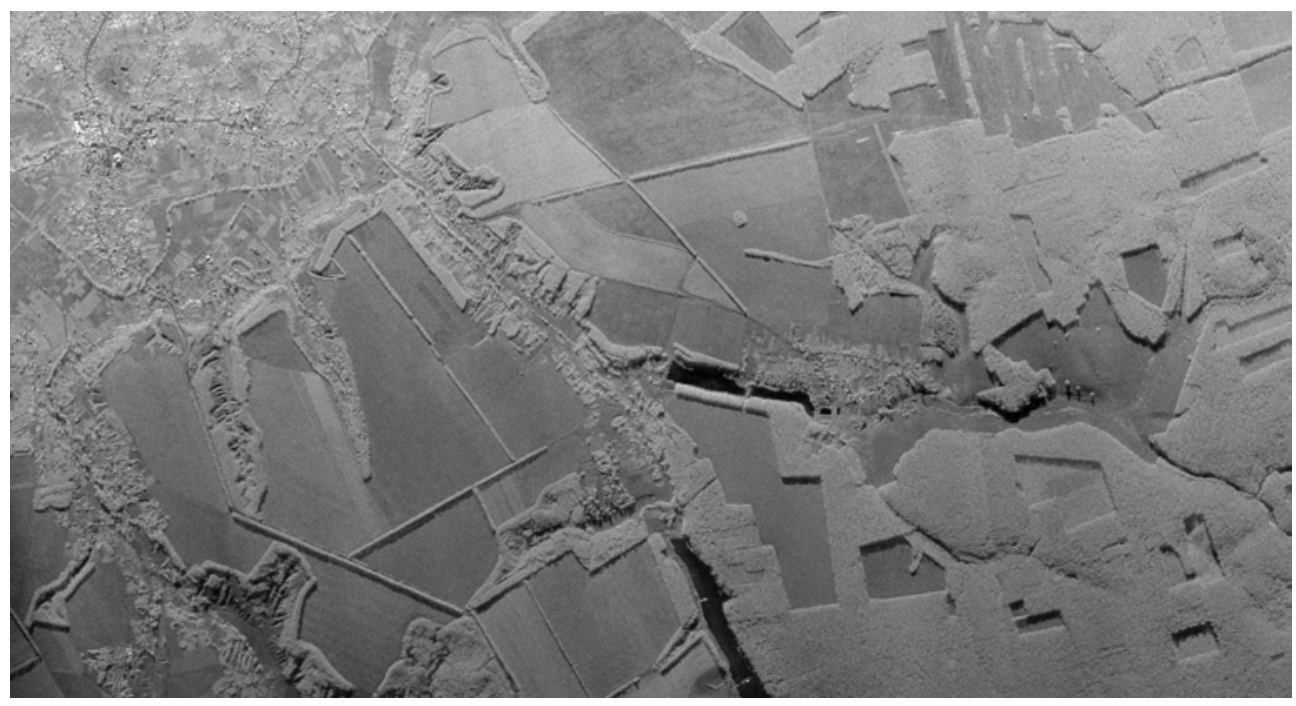

Fig. 17. An example of a 7-look SAR image obtained with the X-band SAR system. 


\section{Practical Ku- and X-band SAR systems}

In this section we describe the design and basic technical characteristics of the mentioned already the $\mathrm{Ku}$ - and X-band SAR systems developed and produced at the Institute of Radio Astronomy of the National Academy of Sciences of Ukraine (Vavriv at al., 2006; Vavriv \& Bezvesilniy, 2011a; Vavriv at al., 2011). The SAR systems were designed to be deployed on a light-weight aircraft. The systems were successfully operated from AN-2 and Y-12 aircrafts.

\subsection{Airborne system RIAN-SAR-Ku}

The Ku-band SAR system RIAN-SAR-Ku Ukraine (Vavriv at al., 2006; Vavriv \& Bezvesilniy, 2011a; Vavriv at al., 2011) operates in a strip-map mode producing single-look SAR images with a 3-meter resolution in real time. The radar can perform measurements at two linear polarizations. The system has also a Motion Target Indication (MTI) capability. Characteristics of the system hardware are listed in Table 1.

\subsubsection{Hardware solutions}

The radar transmitter is based on a traveling-wave tube power amplifier (TWT PA). The radar transmits long pulses with the duration of $5 \mu \mathrm{s}$. The binary phase codding technique is used for the pulse compression to achieve a 3-meter range resolution. The M-sequences of the length of 255 are used for phase codding. The transmitted pulse bandwidth is $50 \mathrm{MHz}$.

A high pulse repetition frequency (PRF) of $20 \mathrm{kHz}$ is required in the system for detection of moving targets. The application of binary phase codding allows us to simplify dramatically the hardware realization of the range compression as compared to the well-known pulse compression technique of pulses with linear frequency modulation (LFM). It is critical to manage the range compression in real time at the high PRF of $20 \mathrm{kHz}$.

The pulse repetition frequency is adjusted continuously to keep the ratio of the aircraft velocity to the PRF constant. It means that the aircraft always flights the same distance during the pulse repetition period. Such approach is used to simplify the further SAR processing.

A sensitive receiver with the noise figure of $2.5 \mathrm{~dB}$ is used in the SAR. The system losses are $4.0 \mathrm{~dB}$. The received data are sampled with two 12-bit ADCs at the sampling frequency of $100 \mathrm{MHz}$.

For the detection of moving targets, we used the following simple principle: All signals, which are detected outside of the Doppler spectrum of the ground echo, are assumed to be signals of moving targets. This approach calls for using of a narrow-beam antenna so that the Doppler spectrum from the ground is narrow. Therefore, a long slotted-waveguide antenna of the length of $1.8 \mathrm{~m}$ with a 1-degree beam has been used. The antenna is actually built of two separate antennas so that the SAR system can operate at two orthogonal linear polarizations.

The usage of such narrow-beam antennas is not common for airborne SAR systems. It imposes the following limitations on SAR imaging.

First, the azimuth resolution in the strip-map mode is limited by the half of the antenna length that is about $1 \mathrm{~m}$ for this system. If we degrade this resolution to $3 \mathrm{~m}$, which is equal to the range resolution, it is possible to build only 5 half-overlapped SAR looks. 


\begin{tabular}{|c|c|c|}
\hline Parameter & RIAN-SAR-Ku & RIAN-SAR-X \\
\hline \multicolumn{3}{|l|}{ Transmitter } \\
\hline Transmitter type & TWT PA* & SSPA** $^{* *}$ \\
\hline Operating frequency & Ku-band & X-band \\
\hline Transmitted peak power & $100 \mathrm{~W}$ & $120 \mathrm{~W}$ \\
\hline Pulse repetition frequency & $5-20 \mathrm{kHz}$ & $3-5 \mathrm{kHz}$ \\
\hline Pulse repetition rate & $<200 \mathrm{~Hz} /(\mathrm{m} / \mathrm{s})$ & Not used \\
\hline Pulse compression technique & $\begin{array}{l}\text { Binary phase codding } \\
\text { (M-sequences) }\end{array}$ & $\begin{array}{l}\text { Linear frequency } \\
\text { modulation }\end{array}$ \\
\hline Pulse bandwidth & $50 \mathrm{MHz}$ & $100 \mathrm{MHz}$ \\
\hline Pulse duration & $5.12 \mu \mathrm{s}$ & $5-16 \mu \mathrm{s}$ \\
\hline \multicolumn{3}{|l|}{ Receiver } \\
\hline Receiver type & Analogue & Digital \\
\hline Receiver bandwidth & $100 \mathrm{MHz}$ & $100 \mathrm{MHz}$ \\
\hline Receiver noise figure & $2.5 \mathrm{~dB}$ & $2.0 \mathrm{~dB}$ \\
\hline System losses & $4.0 \mathrm{~dB}$ & $1.5 \mathrm{~dB}$ \\
\hline ADC sampling frequency & $100 \mathrm{MHz}$ & $200 \mathrm{MHz}$ \\
\hline ADC capacity & 12 bit & 14 bit \\
\hline \multicolumn{3}{|l|}{ Antenna } \\
\hline Antenna type & $\begin{array}{c}\text { Slotted-waveguide / } \\
\text { Horn }\end{array}$ & Slotted-waveguide \\
\hline $\begin{array}{l}\text { Antenna beam width in } \\
\text { azimuth }\end{array}$ & $1^{\circ} / 7^{\circ}$ & $10^{\circ}$ \\
\hline $\begin{array}{l}\text { Antenna beam width in } \\
\text { elevation }\end{array}$ & $40^{\circ} / 40^{\circ}$ & $40^{\circ}$ \\
\hline Antenna gain & $30 \mathrm{~dB} / 21 \mathrm{~dB}$ & $20 \mathrm{~dB}$ \\
\hline Polarization & $\mathrm{HH}$ or VV / VV & VV \\
\hline \multicolumn{3}{|l|}{ SAR Platform } \\
\hline Aircraft flight velocity & $30-80 \mathrm{~m} / \mathrm{s}$ & $30-80 \mathrm{~m} / \mathrm{s}$ \\
\hline Aircraft flight altitude & $1000-5000 \mathrm{~m}$ & $1000-5000 \mathrm{~m}$ \\
\hline Aircrafts used & AN-2, Y-12 & AN-2 \\
\hline
\end{tabular}

* TWT PA is an acronym for a traveling-wave tube power amplifier.

** SSPA is an acronym for a solid-state power amplifier.

Table 1. Characteristics of the SAR hardware systems.

Second, the antenna beam orientation should be measured with a high accuracy of about $0.1^{\circ}$ (that is $1 / 10$ th of the antenna beam width) to avoid radiometric errors in SAR images. The application of the antenna with a wider beam would simplify this requirement. An alternative horn antenna with a 7-degree beam was used to make the system capable of producing high-quality SAR images with many looks by processing of the recorded data.

\subsubsection{Signal processing solutions}

Radar data processing is performed with a special PCI-board equipped with a DSP and an FPGA. Characteristics of the SAR data processing system are given in Table 2. 


\begin{tabular}{|c|c|c|}
\hline Parameter & RIAN-SAR-Ku & RIAN-SAR-X \\
\hline \multicolumn{3}{|l|}{ Range processing } \\
\hline Range resolution & $3 \mathrm{~m}$ & $2 \mathrm{~m}$ \\
\hline Range sampling interval & $1.5 \mathrm{~m}$ & $1.5 \mathrm{~m}$ \\
\hline Number of range gates & 1024 & $\begin{array}{c}2048 \text { (processed) / } 4096 \\
\text { (raw) }\end{array}$ \\
\hline Range swath width & $1536 \mathrm{~m}$ & $3072 \mathrm{~m}$ \\
\hline \multicolumn{3}{|l|}{ Azimuth processing } \\
\hline SAR processing algorithm & $\begin{array}{l}\text { Time-domain convolution } \\
\text { (stream-based) }\end{array}$ & $\begin{array}{l}\text { Range-Doppler algorithm } \\
\text { (frame-based) }\end{array}$ \\
\hline $\begin{array}{l}\text { Real-time motion error } \\
\text { compensation (trajectory) }\end{array}$ & No & $\begin{array}{l}\text { Yes, 1st- and 2nd-order } \\
\text { MOCO }\end{array}$ \\
\hline Clutter-lock* & Line-by-line & Frame-by-frame \\
\hline Pre-filtering & Yes & Yes \\
\hline Azimuth resolution & $3.0 \mathrm{~m}$ & $2.0 \mathrm{~m}$ \\
\hline $\begin{array}{l}\text { Number of looks (in real } \\
\text { time) }\end{array}$ & 1 & $1-15$ \\
\hline $\begin{array}{l}\text { Ground mapping of SAR } \\
\text { images }\end{array}$ & Post-processing & In real time \\
\hline \multicolumn{3}{|l|}{ Data recording } \\
\hline Raw data & $\begin{array}{l}\text { Range-compressed, } \\
\text { 7-times decimated }\end{array}$ & $\begin{array}{l}\text { Uncompressed, } \\
\text { no decimation }\end{array}$ \\
\hline Recorded raw data rate & $12 \mathrm{MB} / \mathrm{s}$ & $80 \mathrm{MB} / \mathrm{s}$ \\
\hline $\begin{array}{l}\text { Pre-filtered data, } \\
\text { navigation data, SAR } \\
\text { images, etc. }\end{array}$ & Yes & Yes \\
\hline \multicolumn{3}{|l|}{ Other capabilities } \\
\hline $\begin{array}{l}\text { Detection and indication of } \\
\text { moving targets }\end{array}$ & Yes & No \\
\hline
\end{tabular}

* Estimation of the antenna beam orientation angles from the backscattered radar data and updating the SAR reference functions.

Table 2. Characteristics of the SAR data processing systems.

The procedure of pre-filtering was implemented to reduce the high input data rate by a coherent accumulation and down-sampling of the data in azimuth from $20 \mathrm{kHz}$ to about 100 $\mathrm{Hz}$ that is determined by the antenna beam width.

The time-domain convolution-based SAR processing algorithm with range migration correction by interpolation is implemented, as described in Section 4. This algorithm forms each pixel of the SAR image with a separate reference function and a migration curve. Therefore, the algorithm works well under unstable flight conditions. The algorithm is fast enough for the operation in real time, if the length of the convolution is not too long. With the narrow-beam antenna and the pre-filtering procedure, this requirement has been satisfied. The SAR processing system is able to build single-look SAR images with 3-meter resolution in real time. The number of range gates is 1024 resulting in 1536-meter range swath width. 
In order to measure accurately the antenna orientation, the algorithm described in Section 4 for the estimation of the antenna orientation angles directly from Doppler frequencies of backscattered radar signals was introduced. The accuracy of the estimation is about $0.1^{\circ}$. The angles are updated about 10 times per second what is sufficient to track fast variations of the antenna beam orientation.

The estimated angles are used to realize the clutter-lock. The pre-filter and the SAR reference functions are updated rapidly to track variations of the antenna orientation, and thus to avoid radiometric errors in SAR images.

The radar system is able to record the range-compressed data at the data rate of about 12 $\mathrm{MB} / \mathrm{s}$ to hard disk drives for post-processing. A 7-times decimation of the input data stream is used to reduce the data rate for recording. The pre-filtered radar data, the navigation data, and SAR images are recorded as well.

\subsection{Airborne system RIAN-SAR-X}

The X-band SAR system RIAN-SAR-X Ukraine (Vavriv \& Bezvesilniy, 2011a; Vavriv at al., 2011) is capable of producing high-quality multi-look SAR images with a 2-meter resolution in real time. The system is designed to operate from light-weight aircraft platforms in sidelooking or squinted strip-map modes. Characteristics of the radar hardware and the signal processing systems are listed in Tables 1 and 2.

\subsubsection{Hardware solutions}

The radar operates in the X-band. The transmitter is based on a modern solid-state power amplifier (SSPA). The peak transmitted power is $120 \mathrm{~W}$. The radar transmits long pulses with a linear frequency modulation. A direct digital synthesizer (DDS) provides frequency sweeping. The pulse duration can be chosen from 5 to $16 \mu \mathrm{s}$. The transmitted pulse bandwidth is $100 \mathrm{MHz}$. It gives the range resolution of $2 \mathrm{~m}$. The pulse repetition frequency is from $3 \mathrm{kHz}$ to $5 \mathrm{kHz}$, and that guarantees an unambiguous data sampling in the azimuth.

A digital receiver technique has been implemented. The noise figure of the receiver is $2 \mathrm{~dB}$. The system losses are $1.5 \mathrm{~dB}$. We have used one 200-MHz ADC with a 14-bit capacity.

The radar uses a compact slotted-waveguide antenna with a 10-degree beam. The wide beam is used, first, to avoid radiometric errors during the formation of SAR images in real time, and, second, to enable building of high-quality SAR images with a large number of looks at a post-processing stage. The antenna is firmly mounted on the aircraft; however it can be installed either into a side-looking or a 40-degree-squinted position.

The SAR system is designed to be operated from a light-weight aircrafts. During test flights, the SAR system was successfully deployed on an AN-2 aircraft. The aircraft flight altitude could be from $1000 \mathrm{~m}$ to $5000 \mathrm{~m}$, and the aircraft flight velocity is expected to be from 30 $\mathrm{m} / \mathrm{s}$ to $80 \mathrm{~m} / \mathrm{s}$. The implemented SAR processing algorithms can operate beyond of these intervals of flight parameters with minor adjustments.

\subsubsection{Signal processing solutions}

A strip-map SAR processing is performed by using a frame-based range-Doppler algorithm with motion compensation, as described in Section 6. The SAR system is capable of 
producing SAR images with a 2-meter resolution formed of up to 15 looks in real time. A scheme with half-overlapped frames is implemented to provide continuous surveillance of the strip without gaps despite of possible motion instabilities.

The SAR navigation system is based on a simple GPS-receiver capable of measuring the aircraft position and the aircraft velocity vector. The measured position is used to link the obtained SAR images to ground maps, and also to know the flight altitude above the ground. The aircraft flight trajectory is integrated from the measured aircraft velocity with a sufficient accuracy to perform the motion compensation. The antenna beam orientation is estimated from Doppler frequencies of the backscattered radar signals. The pitch and yaw antenna orientation angles are used both for motion compensation and for the aperture synthesis. Such angle estimation is a kind of clutter-lock processing allowing to track variations of the antenna beam orientation by adjusting the SAR data processing algorithm from one radar data frame to another.

The signal processing system is divided on two main parts. The first part of the system performs: 1) range compression of LFM pulses combined with the 1st-order motion compensation, 2) calculation of Doppler centroid values for each range gate (by FFT in azimuth) and estimation of the antenna orientation angles, and 3) pre-filtering of the rangecompressed data. This processing is performed in a special PCI board with a DSP and an FPGA.

The second part of the data processing system forms multi-look SAR images by using a range-Doppler algorithm with the 2nd-order motion compensation. This processing is performed on a PC with an Intel Quad Core CPU (the above-mentioned PCI board is installed on this PC). It gives a flexibility in setting the azimuth processing parameters and allows using the developed SAR system as a suitable test-bed for testing new modifications of various frame-based SAR algorithms.

Stitching of the obtained SAR images into a continuous strip map can be performed on a client PC (or a notebook), while viewing the data in real time or offline.

The SAR system is capable of recording the original uncompressed radar data on a solidstate drives organized in a RAID-0 array at the full pulse repetition rate up to $5 \mathrm{kHz}$. These data are stored together with the navigation data (original GPS measurements, integrated trajectories, estimated orientation angles, motion compensation curves, etc.), as well as the pre-filtered range-compressed data and the SAR images formed in real time. Recorded data are used further in our research and development activity on SAR systems.

\section{Conclusion}

The presented results indicate that some of the essential problems that limited the development of SAR systems for small aircrafts are solved. In particular, the problem of the antenna beam orientation evaluation has been solved by extracting this information from the Doppler shift of the radar echoes. This technique enables to use only a simple GPS receiver to provide a reliable SAR operation. Simultaneously, the problem of the correction of the geometrical distortions in SAR images has been solved via the introduction of a signal processing algorithm, which provides pointing multi-look SAR beams exactly to the nodes of a rectangular grid on the ground plane. The proposed multi-look processing algorithm 
with extended number of looks has demonstrated a high efficiency for the correction of radiometric errors. The suggested approaches have been successfully implemented in and tested with $\mathrm{Ku}$ - and X-band SAR systems deployed on small aircrafts. It should be pointed that these solutions are as well useful for SAR systems deployed on other platforms.

\section{Acknowledgment}

The authors would like to thank all of their colleagues at the Department of Microwave Electronics, Institute of Radio Astronomy of the National Academy of Sciences of Ukraine for their help and fruitful discussions. In particular, we indebt to Dr. V. V. Vynogradov, Dr. V. A. Volkov, Dr. S. V. Sosnytskiy, Mr. R. V. Kozhyn, Mr. S. S. Sekretarov, Mr. A. Kravtsov, Mr. A. Suvid, and Mr. I. Gorovyi for their essential contributions to the development of practical SAR systems.

\section{References}

Bamler, R. \& Hartl, P. (1998). Synthetic aperture radar interferometry. Inverse Problems, Vol. 14, pp. R1-R54.

Bezvesilniy, O. O., Dukhopelnykova, I. V., Vynogradov, V. V., \& Vavriv, D. M. (2006). Retrieving 3D relief from radar returns with single-antenna, strip-map airborne SAR. Proceedings of the 6th European Conference on Synthetic Aperture Radar (EUSAR2006). 16-18 May 2006, Dresden, Germany. pp. 1-4. (CD-ROM Proceedings).

Bezvesilniy, O. O., Dukhopelnykova, I. V., Vynogradov, V. V. \& Vavriv, D. M. (2007). Retrieving 3-D topography by using a single-antenna squint-mode airborne SAR. IEEE Transactions on Geoscience and Remote Sensing, Vol. 45, No. 11, pp. 3574-3582.

Bezvesilniy, O. O., Vynogradov, V. V. \& Vavriv, D. M. (2008). High-accuracy Doppler measurements for airborne SAR applications. Proceedings of the 5th European Radar Conference (EuRAD2008). 30-31 Oct. 2008, Amsterdam, The Netherlands. pp. 29-32.

Bezvesilniy, O. O., Gorovyi, I. M., Sosnytskiy, S. V., Vynogradov V. V. \& Vavriv D. M. (2010a). Multi-look stripmap SAR processing algorithm with built-in correction of geometric distortions. Proceedings of the 8th European Conference on Synthetic Aperture Radar (EUSAR2010). 7-10 June 2010, Aachen, Germany. pp. 712-715.

Bezvesilniy, O. O., Gorovyi I. M., Sosnytskiy, S. V., Vynogradov V.V. \& Vavriv D.M. (2010b). Multi-look SAR processing with build-in geometric correction, Proc. of the 11th Int. Radar Symposium (IRS-2010). June 16-18, Vilnius, Lithuania. Vol. 1. pp. 30-33.

Bezvesilniy, O. O., Gorovyi, I. M., Vynogradov V.V. \& Vavriv D.M. (2010c). Correction of radiometric errors by multi-look processing with extended number of looks, Proceedings of the 11th Int. Radar Symposium (IRS-2010). June 16-18, Vilnius, Lithuania. Vol. 1. pp. 26-29.

Bezvesilniy, O. O., Gorovyi, I. M., Sosnytskiy, S. V., Vynogradov, V. V. \& Vavriv, D. M. (2010d). Improving SAR images: Built-in geometric and multi-look radiometric corrections. Proceedings of the 7th European Radar Conference (EuRAD2010). 30 September - 1 October 2010, Paris, France. pp. 256-259.

Bezvesilniy, O. O., Gorovyi, I. M., Sosnytskiy, S. V., Vynogradov, V. V. \& Vavriv, D. M. (2011a). SAR processing algorithm with built-in geometric correction. Radio Physics and Radio Astronomy, Vol. 16, No. 1, pp. 98-108. 
Bezvesilniy, O. O., Gorovyi, I. M., Vynogradov, V. V. \& Vavriv, D. M. (2011b). Multi-look radiometric correction of SAR images. Radio Physics and Radio Astronomy, Vol. 16, No. 4, pp. ???-??? (Accepted for publication).

Bezvesilniy, O. O., Gorovyi, I. M., Vynogradov, V. V. \& Vavriv, D. M. (2011c). RangeDoppler algorithm with extended number of looks, Proceedings of the 2011 Microwaves, Radar and Remote Sensing Symposium (MRRS-2011). August 25-27, Kiev, Ukraine. pp. 203-206.

Blacknell, D., Freeman, A., Quegan, S., Ward, I. A., Finley, I. P., Oliver, C. J., White, R. G. \& J. W. Wood (1989). Geometric accuracy in airborne SAR images. IEEE Transactions on Aerospace and Electronic Systems, Vol. 25, No. 2, pp. 241-258.

Buckreuss, S. (1991). Motion errors in an airborne synthetic aperture radar system. European Transactions on Telecommunications, Vol. 2, No. 6, pp. 655-664.

Carrara, W. G., Goodman, R. S. \& Majewski, R. M. (1995). Spotlight Synthetic Aperture Radar: Signal Processing Algorithms, Artech House, ISBN 0-89006-728-7.

Cumming, I. G. \& Wong, F. H. (2005). Digital Processing of Synthetic Aperture Radar Data: Algorithms and Implementation, Artech House, ISBN 1-58053-058-3.

Franceschetti, G. \& Lanari, R. (1999). Synthetic Aperture Radar Processing, CRC Press, ISBN 08493-7899-0.

Li, F.-K., Held, D. N., Curlander, J. C. \& Wu, C. (1985). Doppler parameter estimation for spaceborne synthetic-aperture radars. IEEE Transactions on Geoscience and Remote Sensing, Vol. 23, No. 1, pp. 47-56.

Madsen, S. N. (1989). Estimating the Doppler centroid of SAR data. IEEE Transactions on Aerospace and Electronic Systems, Vol. 25, No. 2, pp. 134-140.

Moreira, A. (1991). Improved multilook techniques applied to SAR and SCANSAR imagery. IEEE Transactions on Geoscience and Remote Sensing, Vol. 29, No. 4, pp. 529-534.

Oliver, C. J. \& Quegan, S. (1998). Understanding Synthetic Aperture Radar Images, Artech House, ISBN 0-89006-850-X.

Rosen, P. A., Hensley, S., Joughin, I. R., Li, F.-K., Madsen, S. N., Rodriguez, E. \& Goldstein, R. M. (2000). Synthetic aperture radar interferometry. Proceedings of the IEEE, Vol. 88, No. 3, pp. 333-382.

Vavriv, D. M., Vynogradov, V. V., Volkov, V. A., Kozhyn, R. V., Bezvesilniy, O. O., Alekseenkov, S. V., Shevchenko, A. V., Belikov, A., Vasilevsky, M.P. \& Zaikin D. I. (2006). Cost-effective airborne SAR. Radio Physics and Radio Astronomy, Vol. 11, No. 3, pp. 276-297.

Vavriv, D. M. \& Bezvesilniy, O. O. (2011a). Developing SAR for small aircrafts in Ukraine. Proceedings of the 2011 IEEE MTT-S International Microwave Symposium (IMS 2011). 510 June 2011, Baltimore, USA. pp. 1-4. (CD-ROM Proceedings).

Vavriv, D. M. \& Bezvesilniy, O. O. (2011b). Potential of multi-look SAR processing. Proceedings of the 5th Int. Conference on Recent Advances in Space Technologies (RAST 2011). 9-11 June 2011, Istanbul, Turkey. pp. 365-369.

Vavriv, D. M., Bezvesilniy, O. O., Kozhyn, R. V., Vynogradov, V. V., Volkov, V. A. \& Sekretarov, S. S. (2011). SAR systems for light-weight aircrafts. Proceedings of the 2011 Microwaves, Radar and Remote Sensing Symposium (MRRS-2011). August 25-27, Kiev, Ukraine. pp. 15-19.

Wehner, D.R. (1995). High-Resolution Radar (2nd Ed.), Artech House, ISBN 0-89006-727-9. 


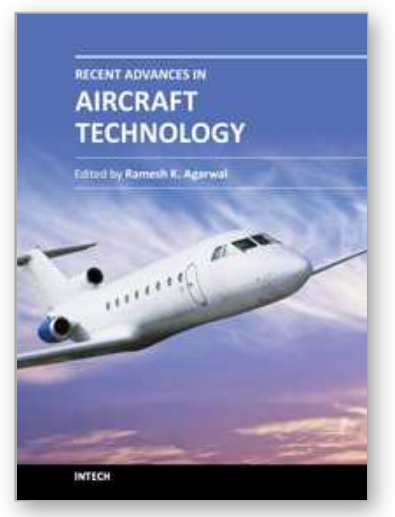

\author{
Recent Advances in Aircraft Technology \\ Edited by Dr. Ramesh Agarwal
}

ISBN 978-953-51-0150-5

Hard cover, 544 pages

Publisher InTech

Published online 24, February, 2012

Published in print edition February, 2012

The book describes the state of the art and latest advancements in technologies for various areas of aircraft systems. In particular it covers wide variety of topics in aircraft structures and advanced materials, control systems, electrical systems, inspection and maintenance, avionics and radar and some miscellaneous topics such as green aviation. The authors are leading experts in their fields. Both the researchers and the students should find the material useful in their work.

\title{
How to reference
}

In order to correctly reference this scholarly work, feel free to copy and paste the following:

Oleksandr O. Bezvesilniy and Dmytro M. Vavriv (2012). Synthetic Aperture Radar Systems for Small Aircrafts: Data Processing Approaches, Recent Advances in Aircraft Technology, Dr. Ramesh Agarwal (Ed.), ISBN: 978953-51-0150-5, InTech, Available from: http://www.intechopen.com/books/recent-advances-in-aircrafttechnology/synthetic-aperture-radar-systems-for-small-aircrafts-data-processing-approaches

\section{INTECH}

open science | open minds

\author{
InTech Europe \\ University Campus STeP Ri \\ Slavka Krautzeka 83/A \\ 51000 Rijeka, Croatia \\ Phone: +385 (51) 770447 \\ Fax: +385 (51) 686166 \\ www.intechopen.com
}

\author{
InTech China \\ Unit 405, Office Block, Hotel Equatorial Shanghai \\ No.65, Yan An Road (West), Shanghai, 200040, China \\ 中国上海市延安西路65号上海国际贵都大饭店办公楼 405 单元 \\ Phone: +86-21-62489820 \\ Fax: $+86-21-62489821$
}


(C) 2012 The Author(s). Licensee IntechOpen. This is an open access article distributed under the terms of the Creative Commons Attribution 3.0 License, which permits unrestricted use, distribution, and reproduction in any medium, provided the original work is properly cited. 